\title{
A Reactivity/Affinity Switch for Parallel Kinetic Resolution: $\alpha$ - Amino Acid Quasienantiomers and the Resolution of Cyclopropene Carboxylic Acids
}

\author{
Lian-an Liao, Fan Zhang, Olga Dmintrenko, Robert D. Bach and Joseph M. Fox* \\ Brown Laboratories, Department of Chemistry and Biochemistry, University of \\ Delaware, Newark, DE 19716.
}

\section{Supporting Information Experimental Section}

General Considerations : All reactions were carried out in glassware that was flamedried under vacuum, and cooled under nitrogen. THF was distilled under nitrogen from $\mathrm{Na}$ /benzophenone. Triethylamine was distilled under nitrogen from $\mathrm{CaH}_{2}$. 1 Adamantoyl chloride was prepared from 1-adamantane carboxylic acid and thionyl chloride. The other reagents were purchased from commercial sources. (S)phenyloxazolidione was purchased from Chemicrea Inc., Tokyo, JAPAN. Chromatography was performed on silica gel (ICN SiliTech $32-62 \mathrm{D}, 60 \AA$ ). For ${ }^{13} \mathrm{C}$ NMR, multiplicities were distinguished using an APT pulse sequence: typical methylene and quaternary carbons appear 'up' (u); methane and methyl carbons 'down' (dn). Exceptions are methane carbons of cyclopropenes, which usually have the same phase as 'normal' methylenes and quaternary carbons. Diastereomer purities were determined using normal phase silica HPLC columns, and enantiomeric excesses were determined by HPLC using a CHIRACEL OD column. Yields in Tables refer to isolated yields (average of 2 runs) of compounds estimated to be $>95 \%$ pure as determined by HPLC and ${ }^{1} \mathrm{H}$ NMR.

\section{Synthesis of 4(R)-(4-t-butyldimethylsilyloxyphenyl)oxazolidinone (6R)}

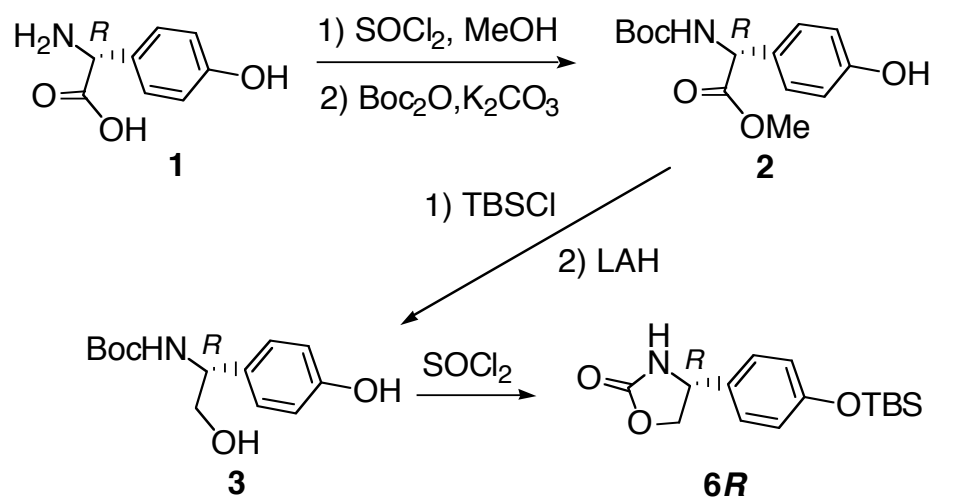

(R)-N-(t-butoxycarbonyl)-4-hydroxyphenylglycine methyl ester (2) A $500 \mathrm{~mL}$ round bottomed flask containing (D)-4-hydroxylphenylglycine (1) $(15.10 \mathrm{~g}, 90.42 \mathrm{mmol})$ in 100 $\mathrm{mL}$ methanol was cooled in a bath of ice water. Thionyl chloride $(32.62 \mathrm{~g}, 281.2 \mathrm{mmol})$ 
was dropwise via additional funnel. The reaction mixture was refluxed overnight and then cooled to rt. Solvents were removed under reduced pressure (using an aspirator) to give $(R)$-4-hydroxyphenylglycine methyl ester as white solid that was used in the next step without purification.

This white solid was obtained was dissolved in $200 \mathrm{~mL}$ of dioxane $/ \mathrm{H}_{2} \mathrm{O}(1: 1)$ and cooled in an ice bath. $\mathrm{K}_{2} \mathrm{CO}_{3}(50.0 \mathrm{~g}, 361.68 \mathrm{mmol})$ and $\mathrm{Boc}_{2} \mathrm{O}(21.34 \mathrm{~g}, 94.94 \mathrm{mmol})$ were added successively. After the mixture was stirred at $\mathrm{rt}$ for $4 \mathrm{hrs}, 250 \mathrm{~mL}$ of $0.3 \mathrm{M} \mathrm{HCl}(\mathrm{aq})$ was added. After extracting with ethyl acetate $(4 \times 50 \mathrm{~mL})$ and washing with brine, the organics were dried over $\mathrm{Na}_{2} \mathrm{SO}_{4}$, filtered and concentrated to give 2 (25.6 g crude weight). The crude material was directly used in next reaction without purification. An analytical sample was obtained through purification by flash chromatography on silica gel (10\% ethyl acetate in hexanes). mp 138.0-138.5 ${ }^{\circ} \mathrm{C} .{ }^{1} \mathrm{H} \mathrm{NMR}\left(400 \mathrm{MHz}, \mathrm{CDCl}_{3}, \delta\right)$ : $7.15(\mathrm{~d}, J=8.1 \mathrm{~Hz}, 2 \mathrm{H}), 7.14(\mathrm{bs}, 1 \mathrm{H}), 6.72(\mathrm{~d}, J=8.5 \mathrm{~Hz}, 2 \mathrm{H}), 5.65-5.40(\mathrm{~m}, 1 \mathrm{H})$, 5.21-5.00 (m, 1H), 3.69 (s, 3H), 1.43 (bs, 9H); $\left.{ }^{13} \mathrm{C} \mathrm{NMR} \mathrm{(100MHz,} \mathrm{CDCl}_{3}, \delta\right) 172.5(\mathrm{u})$, 156.9 (u), 155.6 (u), 128.8 (dn), 128.3 (u), $116.3(\mathrm{dn}), 81.1$ (u), 57.5 (dn), 53.2 (dn), 28.7 (dn); IR (neat, $\mathrm{cm}^{-1}$ ) 3431, 3364, 1725, 1669, 1614, 1504, 1443, 1320, 1163, 1060, 996, 835; LC-MS (ESI+) [M+Na]: 304.1.

$(R)-\mathrm{N}$-(t-butoxycarbonyl)-4-(t-butyldimethylsiloxy)phenylglycine methyl ester To a $500 \mathrm{~mL}$ round bottomed flask containing a solution of crude $2(25.3 \mathrm{~g})$ in $250 \mathrm{~mL} \mathrm{CH}_{2} \mathrm{Cl}_{2}$ was added tert-butyldimethylsilyl chloride (14.92 g, $94.5 \mathrm{mmol})$, triethylamine $(13.64 \mathrm{~g}$, $135.0 \mathrm{mmol})$ and DMAP $(1.10 \mathrm{~g}, 9.0 \mathrm{mmol})$. The mixture was refluxed at $50^{\circ} \mathrm{C}$ for $5 \mathrm{~h}$, cooled to rt, and then sequentially washed with $150 \mathrm{~mL}$ of $0.3 \mathrm{M} \mathrm{HCl}(\mathrm{aq}), 100 \mathrm{~mL}$ of $\mathrm{H}_{2} \mathrm{O}$, and $100 \mathrm{~mL}$ of brine. The organics were dried over $\mathrm{Na}_{2} \mathrm{SO}_{4}$ and concentrated to give $41.5 \mathrm{~g}$ crude $(R)$ - $\mathrm{N}$-( $t$-butoxycarbonyl)-4-( $t$-butyldimethylsiloxy)phenylglycine methyl ester. The crude material was directly used in next reaction without purification. An analytical sample was obtained through purification by flash chromatography on silica gel (10\% ethyl acetate in hexanes). The product is a semi-solid. ${ }^{1} \mathrm{H}$ NMR (400MHz, $\left.\mathrm{CDCl}_{3}, \delta\right): 7.19(\mathrm{~d}, J=8.5,2 \mathrm{H}), 6.78(\mathrm{~d}, J=8.5 \mathrm{~Hz}, 2 \mathrm{H}), 5.49-5.25(\mathrm{~m}, 1 \mathrm{H})$, 5.22-4.95 (m, 1H), $3.68(\mathrm{~s}, 3 \mathrm{H}), 1.40(\mathrm{bs}, 9 \mathrm{H}), 0.95(\mathrm{~s}, 9 \mathrm{H}), 0.17(\mathrm{~s}, 6 \mathrm{H}) ;{ }^{13} \mathrm{C} \mathrm{NMR}$ $\left(100 \mathrm{MHz}, \mathrm{CDCl}_{3}, \delta\right) 172.4(\mathrm{u}), 156.2(\mathrm{u}), 155.3(\mathrm{u}), 129.8(\mathrm{u}), 128.8(\mathrm{dn}), 120.8(\mathrm{dn})$, $80.4(\mathrm{u}), 57.5(\mathrm{dn}), 53.0(\mathrm{dn}), 28.7(\mathrm{dn}), 26.0(\mathrm{dn}), 18.6(\mathrm{u}),-4.0(\mathrm{dn})$; IR (neat, $\left.\mathrm{cm}^{-1}\right)$ 2957, 2860, 1746, 1712, 1608, 1509, 1392, 1367, 1264, 1166, 1055, 1028, 912, 840, 807, 782, 734; LC-MS (ESI+) [M+Na]: 418.2.

(R)-N-(t-butoxycarbonyl)-4-(t-butyldimethylsiloxy)phenylglycinol (3) A 1 L round bottomed flask charged with a solution of crude $(R)-\mathrm{N}-(t$-butoxycarbonyl)-4- $(t$ butyldimethylsiloxy)phenylglycine methyl ester in $500 \mathrm{~mL}$ THF. The solution was cooled in an ice bath, and lithium aluminum hydride $(10.8 \mathrm{~g}, 270 \mathrm{mmol})$ was added slowly and in portions. The mixture was stirred for $1.5 \mathrm{hrs}$, and then quenched by careful addition of sat. $\mathrm{NH}_{4} \mathrm{Cl}(\mathrm{aq})$. After the quenching was completed, $100 \mathrm{~mL}$ of $0.3 \mathrm{M} \mathrm{HCl}$ was added. The mixture was then filtered through celite, and the filtered residue was rinsed with ether. The filtrate was extracted with ether $(3 \times 100 \mathrm{~mL})$. The organics were combined and washed with brine, dried over $\mathrm{Na}_{2} \mathrm{SO}_{4}$ and concentrated to give crude alcohol 3. An analytical sample was obtained through purification by flash 
chromatography on silica gel (20\% ethyl acetate in hexanes). The product is a semisolid. ${ }^{1} \mathrm{H}$ NMR (400MHz, $\left.\mathrm{CDCl}_{3}, \delta\right): 7.14(\mathrm{~d}, J=8.5 \mathrm{~Hz}, 2 \mathrm{H}), 6.81(\mathrm{~d}, J=8.5 \mathrm{~Hz}, 2 \mathrm{H}), 5.15$ (bs, 1H), 4.72 (bs, 1H), 3.80(m, 2H), 2.50 (bs, 1H), 1.43 (bs, 9H), 0.97 (s, 9H), 0.18 (s, $6 \mathrm{H}) ;{ }^{13} \mathrm{C}$ NMR $\left(100 \mathrm{MHz}, \mathrm{CDCl}_{3}, \delta\right) 156.2,155.2,131.8,127.7,120.3,80.0,67.2,56.5$, 28.3, 25.6, 18.2, -4.4; IR (neat, $\mathrm{cm}^{-1}$ ) 2932, 2860, 2360, 2339, 1692, 1609, 1510, 1471, 1391, 1366, 1251, 1166, 1102, 1053, 912, 837, 803, 779; LC-MS (ESI+) [M+Na]: 390.2.

4(R)-(4-t-butyldimethylsilyloxyphenyl)oxazolidinone $(6 R)$ A 1 L round bottomed flask containing a solution of crude $\mathbf{3}$ in $400 \mathrm{~mL}$ THF was cooled in an ice-water bath. Thionyl chloride $(86.7 \mathrm{~g}, 720 \mathrm{mmol})$ was added dropwise via additional funnel. The mixture was stirred at rt overnight. Excess $\mathrm{SOCl}_{2}$ was removed by distillation, and the residue was pumped to dryness using an aspirator. The resulting solid was dissolved in $200 \mathrm{~mL}$ ethyl acetate and sequentially washed with water, sat. $\mathrm{NaHCO}_{3}$ and brine. The organics were dried over $\mathrm{Na}_{2} \mathrm{SO}_{4}$ and concentrated. $\mathrm{CH}_{2} \mathrm{Cl}_{2}(200 \mathrm{~mL})$ was added to the residue, and an insoluble solid (4(R)-(4-hydroxyphenyl)oxazolidinone (28) was removed by filtration. The filtrate was concentrated, dissolved in ca. $100 \mathrm{~mL}$ of hot ethyl acetate, and cooled to rt. Hexane (ca. $100 \mathrm{~mL}$ ) was added until the cloud point to induce crystallization. After a recrystallation from ethyl acetate/hexane, the two crops gave a combined yield of 10.80 $\mathrm{g}$ of a light yellow solid. Chromatography of the mother liquor (20\% ethyl acetate in hexanes) gave an additional 5.02g. The total yield from 1 was $16.82 \mathrm{~g}(60 \%)$. $\mathrm{mp}: 142-$ $142.5^{\circ} \mathrm{C} ;[\alpha]_{\mathrm{D}}:-37.6$ (c 1.05, THF); ${ }^{1} \mathrm{H}$ NMR $\left(\mathrm{CDCl}_{3}, 400 \mathrm{MHz}, \delta\right): 7.16-7.18(\mathrm{~m}, 2 \mathrm{H})$, 6.82-6.84 (m, 2H), 6.77(s, $1 \mathrm{H}), 4.87(\mathrm{t}, J=7.8 \mathrm{~Hz}, 1 \mathrm{H}), 4.65(\mathrm{t}, J=8.6 \mathrm{~Hz}, 1 \mathrm{H}), 4.10(\mathrm{q}$, $J=7.1,8.5 \mathrm{~Hz}, 1 \mathrm{H}) 0.97(\mathrm{~s}, 9 \mathrm{H}), 0.19(\mathrm{~s}, 6 \mathrm{H}) ;{ }^{13} \mathrm{C} \mathrm{NMR}\left(\mathrm{CDCl}_{3}, 90 \mathrm{MHz}, \delta\right): 160.1(\mathrm{u})$, 155.8(u), 132.1(u), 127.1(dn), 120.4(dn), 72.6(u), 55.8(dn), 25.5(dn), 18.0(u), -4.6(dn); IR(neat, $\mathrm{cm}^{-1}$ ) 2929, 2857, 1731, 1713, 1612, 1510, 1249, 1172, 1100, 1029, 920, 832; HRMS-CI $\left(\mathrm{NH}_{3}\right) \mathrm{m} / \mathrm{z}$ : [M+] calcd for $\mathrm{C}_{15} \mathrm{H}_{23} \mathrm{O}_{3} \mathrm{NSi}$, 293.1447; found, 293.1438.

\section{Synthesis of cyclopropene carboxylic acids}
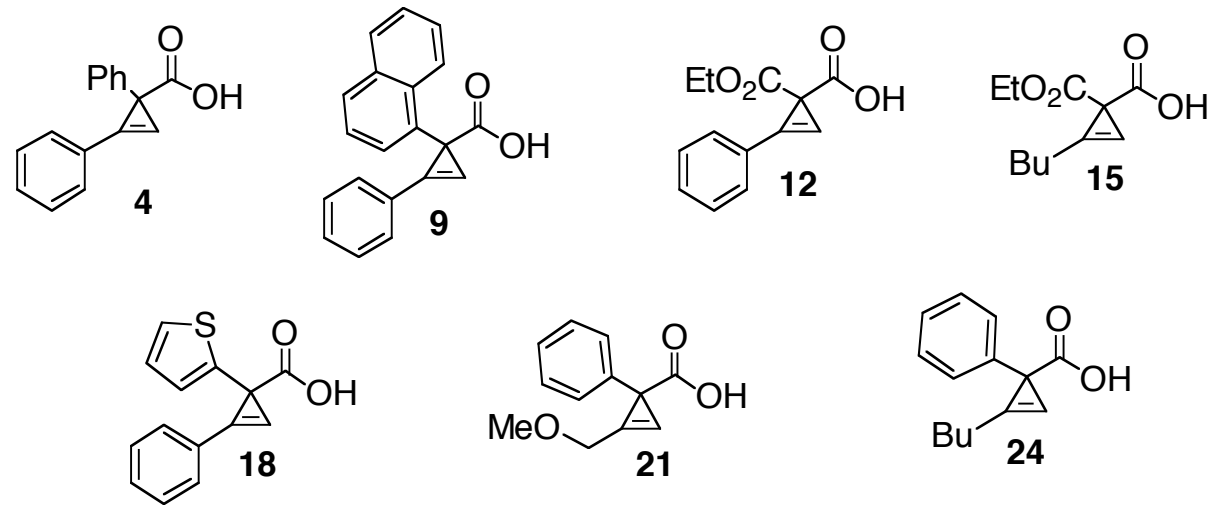

Procedures for the carboxylic acids 4, 9 and 24 have been described previously,

2-Butylcycloprop-2-ene-1,1-dioic acid monoethyl ester (15) A solution of $\mathrm{KOH}$ $(1.72 \mathrm{~g}, 30.71 \mathrm{mmol})$ in $20 \mathrm{~mL}$ water was added dropwise to an solution of diethyl 2butylcycloprop-2-ene-1,1-dicarboxylate ${ }^{2}(7.07 \mathrm{~g}, 29.46 \mathrm{mmol})$ in $75 \mathrm{~mL}$ ethanol that had been cooled in an ice bath. The reaction mixture was allowed to stir at rt for $2 \mathrm{~d}$, at which 
point it was concentrated to remove EtOH and diluted with water. Three extractions with ether $(3 \times 10 \mathrm{~mL})$ removed unchanged diethyl 2-butylcycloprop-2-ene-1,1dicarboxylate. The remaining aqueous layer was acidified with $10 \% \mathrm{HCl}$ (aq) and extracted with ether $(3 \times 20 \mathrm{~mL})$. The organics were combined, sequentially washed with water and brine, dried over $\mathrm{Na}_{2} \mathrm{SO}_{4}$, filtered and concentrated to give the title product $(4.38 \mathrm{~g}, 70 \%)$ as a pale yellow oil. A similar experiment that started with $2.14 \mathrm{~g}$ of diethyl 2-butylcycloprop-2-ene-1,1-dicarboxylate gave $1.43 \mathrm{~g}$ of the title product in $75 \%$ yield. ${ }^{1} \mathrm{H} \mathrm{NMR}\left(\mathrm{CDCl}_{3}, 400 \mathrm{MHz}, \delta\right) 6.17(\mathrm{t}, J=1.4 \mathrm{~Hz}, 1 \mathrm{H}), 4.22(\mathrm{~m}, 2 \mathrm{H}), 2.53(\mathrm{~m}, 2 \mathrm{H})$, $1.58(\mathrm{~m}, 2 \mathrm{H}), 1.42(\mathrm{~m}, 2 \mathrm{H}), 1.27(\mathrm{t}, J=7.1 \mathrm{~Hz}, 3 \mathrm{H}), 0.93(\mathrm{t}, J=7.3 \mathrm{~Hz}, 3 \mathrm{H}) ;{ }^{13} \mathrm{C}$ $\mathrm{NMR}\left(\mathrm{CDCl}_{3}, 100 \mathrm{MHz}, \delta\right) 178.0(\mathrm{u}), 173.1(\mathrm{u}), 110.4(\mathrm{u}), 89.7(\mathrm{u}), 63.0(\mathrm{u}), 31.2(\mathrm{u})$, 28.8 (u), 23.7 (u), 22.5 (u), 14.4 (dn), 14.0 (dn); IR(neat, $\left.\mathrm{cm}^{-1}\right)$ 3137, 2960, 2936, 2875, 1757, 1729, 1694, 1665, 1650, 1416, 1372, 1350, 1061, 851, 714; HRMS-CI(NH$\left.)_{3}\right) \mathrm{m} / \mathrm{z}$ : $[\mathrm{M}+\mathrm{H}]$ calcd for $\mathrm{C}_{11} \mathrm{H}_{16} \mathrm{O}_{4}: 213.1127$, found: 213.1118 .

2-Phenylcycloprop-2-ene-1,1-dioic acid monoethyl ester (12). The procedure was identical to that used to prepared 2-butylcycloprop-2-ene-1,1-dioic acid monoethyl ester (15), except that diethyl 2-phenylcycloprop-2-ene-1,1-dicarboxylate ${ }^{2}(0.95 \mathrm{~g}, 3.65 \mathrm{mmol})$ and $\mathrm{KOH}(0.20 \mathrm{~g}, 3.65 \mathrm{mmol})$ were used. This protocol gave $0.69 \mathrm{~g}(81 \%)$ of the title compound as a pale yellow oil. A similar experiment that started with $0.97 \mathrm{~g}$ of diethyl 2phenylcycloprop-2-ene-1,1-dicarboxylate gave $0.67 \mathrm{~g}(78 \%)$ of the title product. ${ }^{1} \mathrm{H}$ NMR $\left(\mathrm{CDCl}_{3}, 400 \mathrm{MHz}, \delta\right) 7.51(\mathrm{~m}, 2 \mathrm{H}), 7.44(\mathrm{~m}, 2 \mathrm{H}), 6.75(\mathrm{~s}, 1 \mathrm{H}), 4.21(\mathrm{~m}, 2 \mathrm{H}), 1.19(\mathrm{t}$, $J=7.2 \mathrm{~Hz}, 3 \mathrm{H}) ;{ }^{13} \mathrm{C} \mathrm{NMR}\left(\mathrm{CDCl}_{3}, 100 \mathrm{MHz}, \delta\right) 177.1(\mathrm{u}), 172.4(\mathrm{u}), 131.4(\mathrm{dn}), 130.7$ (dn), $129.5(\mathrm{dn}), 123.0(\mathrm{u}), 108.5(\mathrm{u}), 92.3(\mathrm{u}), 63.2(\mathrm{u}), 31.3(\mathrm{u}), 14.4(\mathrm{dn})$; IR(neat, $\left.\mathrm{cm}^{-1}\right)$ 2984, 1730, 1666, 1415, 1372, 1308, 1266, 1062, 892, 732, 694; HRMS-CI(NH $\mathrm{NH}_{3}$ m/z: $[\mathrm{M}+\mathrm{H}]$ calcd for $\mathrm{C}_{13} \mathrm{H}_{13} \mathrm{O}_{4}: 233.0814$, found: 233.0804 .

2-Phenyl-1-(2-thienyl)cycloprop-2-ene-1-carboxylic acid (18): Sequentially added to a dry $100 \mathrm{~mL}$ round bottomed flask were dirhodium tetracetate $(26 \mathrm{mg}, 0.06 \mathrm{mmol})$ and phenylacetylene $(2.0 \mathrm{~g}, 20 \mathrm{mmol})$. A solution of ethyl $\alpha$-(2-thienyl)diazoacetate $(1.0 \mathrm{~g}$, $5.0 \mathrm{mmol}$ ) in $20 \mathrm{~mL} \mathrm{CH} \mathrm{Cl}_{2}$ was added to the mixture via syringe pump at a rate of 0.50 $\mathrm{mL} /$ hour. After the addition was completed, the mixture was allowed to continue stirring for $1 \mathrm{~h}$. The mixture was filtered through a short bed of silica gel and the filtrate was concentrated. The residue was dissolved in $20 \mathrm{~mL}$ of methanol and transferred to a $100 \mathrm{~mL}$ round bottomed flask. $\mathrm{KOH}(25 \mathrm{~mL}$ of $8.5 \%$ aqueous solution) was added and the mixture was stirred overnight at $\mathrm{rt}$. The methanol was removed under reduced pressure, and conc. $\mathrm{HCl}$ was added until $\mathrm{pH} \sim 1-2$. After three extractions with $\mathrm{CH}_{2} \mathrm{Cl}_{2}$, the organics were combined, dried $\left(\mathrm{Na}_{2} \mathrm{SO}_{4}\right)$, filtered, and concentrated. The residue was chromatographed on silica gel (20-40\% ethyl acetate in hexane) to provide $317 \mathrm{mg}(26 \%)$ of 18 - a pale yellow semi-solid. ${ }^{1} \mathrm{H}$ NMR $\left(\mathrm{CDCl}_{3}, 400 \mathrm{MHz}, \delta\right): 7.60-7.62(\mathrm{~m}, 2 \mathrm{H})$, 7.42-7.44 (m, 3H), 7.14-7.16 (dd, J=1.3, 5.1Hz, 1H), 7.09 (s, 1H), 7.01-7.02 (dd, J=1.2, $3.5 \mathrm{~Hz}, 1 \mathrm{H}), 6.90-6.92$ (q, J=3.6, $1.5 \mathrm{~Hz}, 1 \mathrm{H}) ;{ }^{13} \mathrm{C} \mathrm{NMR}\left(\mathrm{CDCl}_{3}, 100 \mathrm{MHz}, \delta\right): 180.1(\mathrm{u})$, 144.7(u), 130.3(dn), 130.2(dn), 128.9(dn), 126.5(dn), 125.2(dn), 124.3(dn), 123.9(u), 114.9(u), 98.3(u), 29.3(u); IR( KBr, $\left.\mathrm{cm}^{-1}\right)$ : 3560, 3031, 2865, 1785, 1601, 1500, 1409, 1222, 1099; HRMS-CI( $\left.\mathrm{NH}_{3}\right) \mathrm{m} / \mathrm{z}$ : [M+], calcd for $\mathrm{C}_{14} \mathrm{H}_{10} \mathrm{O}_{2} \mathrm{~S}, 242.0401$; found, 242.0390 . 
2-Phenyl-1-(methoxymethyl)cycloprop-2-ene-1-carboxylic acid (21) Sequentially added to a dry $100 \mathrm{~mL}$ round bottomed flask were dirhodium tetracetate $56 \mathrm{mg}(0.12 \mathrm{mmol})$ and methyl propargyl ether $4.8 \mathrm{~g}(68 \mathrm{mmol})$. A solution of methyl $\alpha$ -

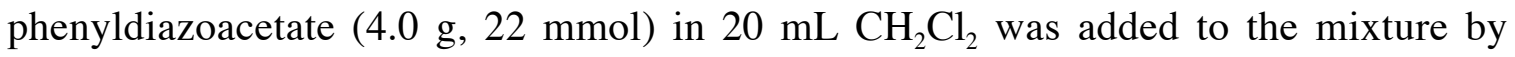
syringe pump at a rate of $0.50 \mathrm{~mL} / \mathrm{h}$. After the addition was completed, the mixture was allowed to continue stirring for $1 \mathrm{~h}$. The mixture was filtered through a short bed of silica gel and the filtrate was concentrated. The residue was dissolved in $50 \mathrm{~mL}$ of methanol and transferred to a $250 \mathrm{~mL}$ round bottomed flask. $\mathrm{KOH}(100 \mathrm{~mL}$ of $8.5 \%$ aqueous solution) was added and the mixture was stirred at $\mathrm{rt}$ overnight. The methanol was removed under reduced pressure, and conc. $\mathrm{HCl}$ was added until $\mathrm{pH} \sim 1-2$. After three extractions with $\mathrm{CH}_{2} \mathrm{Cl}_{2}$, the organics were combined, dried $\left(\mathrm{Na}_{2} \mathrm{SO}_{4}\right)$, filtered, and concentrated. The residue was chromatographed on silica gel (20-40\% ethyl acetate in hexane) to provide $2.50 \mathrm{~g}(54 \%)$ of $\mathbf{2 1}$, a colorless oil. ${ }^{1} \mathrm{H} \mathrm{NMR}\left(\mathrm{CDCl}_{3}, 400 \mathrm{MHz}, \delta\right)$ : 7.28-7.33 (m, 4H), 7.22-7.25 (m, 1H), $6.99(\mathrm{t}, \mathrm{J}=1.6 \mathrm{~Hz}, 1 \mathrm{H}), 4.47-4.58(\mathrm{~m}, 2 \mathrm{H}), 3.40(\mathrm{~s}$, $3 \mathrm{H}) ;{ }^{13} \mathrm{C}$ NMR $\left(\mathrm{CDCl}_{3}, 100 \mathrm{MHz}, \delta\right): 181.2(\mathrm{u}), 139.9$ (u), $128.4(\mathrm{dn}), 128.1(\mathrm{dn}), 126.8$ (dn), $117.0(\mathrm{u}), 100.2(\mathrm{u}), 65.3(\mathrm{u}), 58.7(\mathrm{dn}), 33.4(\mathrm{u}) ; \operatorname{IR}\left(\mathrm{KBr}, \mathrm{cm}^{-1}\right)$ : 3503, 3017, 2869, 1787, 1596, 1500, 1406, 1095; HRMS-CI $\left(\mathrm{NH}_{3}\right) \mathrm{m} / \mathrm{z}$ : [M+], calcd for $\mathrm{C}_{12} \mathrm{H}_{12} \mathrm{O}_{3}$, 204.0217; found, 204.0206.

\section{General Procedures for Parallel Kinetic Resolution.}

To a $50 \mathrm{~mL}$ round bottomed flask was added $(S)$-4-phenyloxazolidinone $(55 \mathrm{mg}, 0.33$ $\mathrm{mmol})$ and $(R)-4$-[4-( $t$-butyldimethylsiloxy)phenyl]oxazolidinone $(97 \mathrm{mg}, 0.33 \mathrm{mmol})$. THF $(10 \mathrm{~mL})$ was added, and the mixture was stirred and cooled in a bath at $-78^{\circ} \mathrm{C}$ (dry ice/acetone). $n$-BuLi $(0.66 \mathrm{mmol}, 0.33 \mathrm{~mL}$ of a $2.0 \mathrm{M}$ pentane solution) was then added via syringe, and the mixture was stirred magnetically for $30 \mathrm{~min}$ at $-78{ }^{\circ} \mathrm{C}$. This solution of lithiated oxazolidinones was then used as indicated below.

A separate $100 \mathrm{~mL}$ round bottomed flask containing the cycloprop-2-ene-1-carboxylic acid $(0.60 \mathrm{mmol})$ and $30 \mathrm{~mL}$ THF was cooled in a bath at $-30^{\circ} \mathrm{C}$ [dry ice $/ 30 \%$ ethylene glycol in ethanol]. The mixture was stirred magnetically under nitrogen atmosphere. Freshly distilled triethylamine $(212 \mathrm{mg}, 0.30 \mathrm{~mL}, 2.1 \mathrm{mmol})$ and adamantanecarbonyl chloride $(125 \mathrm{mg}, 0.63 \mathrm{mmol})$ were added sequentially, and stirring at $-30{ }^{\circ} \mathrm{C}$ was continued for $1 \mathrm{~h}$. The mixture was then cooled in a bath at $-98^{\circ} \mathrm{C}$ (liquid $\mathrm{N}_{2} /$ methanol). To this solution was added via syringe the solution of the lithiated oxazolidiones (prepared above). The mixture was allowed to stir for $2 \mathrm{~h}$ at $-98^{\circ} \mathrm{C}$, and the temperature was allowed to rise to $0{ }^{\circ} \mathrm{C}$. TBAF $(0.5 \mathrm{mmol}, 0.5 \mathrm{~mL}$ of $1.0 \mathrm{M}$ solution in THF solution $)$ was then added, and the mixture stirred for $15 \mathrm{~min}$. The reaction mixture was then quenched with $\mathrm{NH}_{4} \mathrm{Cl}(\mathrm{aq})$, and the solvents were removed under reduced pressure. The residue was partitioned between $50 \mathrm{~mL}$ of ether and $50 \mathrm{~mL}$ of water. The aqueous layer was extracted twice with ether, and the combined organics were dried $\left(\mathrm{Na}_{2} \mathrm{SO}_{4}\right)$, filtered, and concentrated. The residue was chromatographed on silica gel (with a gradient of 15$35 \%$ ethyl acetate in hexane) to provide the quasienantiomeric products. 
Parallel kinetic resolution of 1,2-diphenylcycloprop-2-ene-1-carboxylic acid (4).

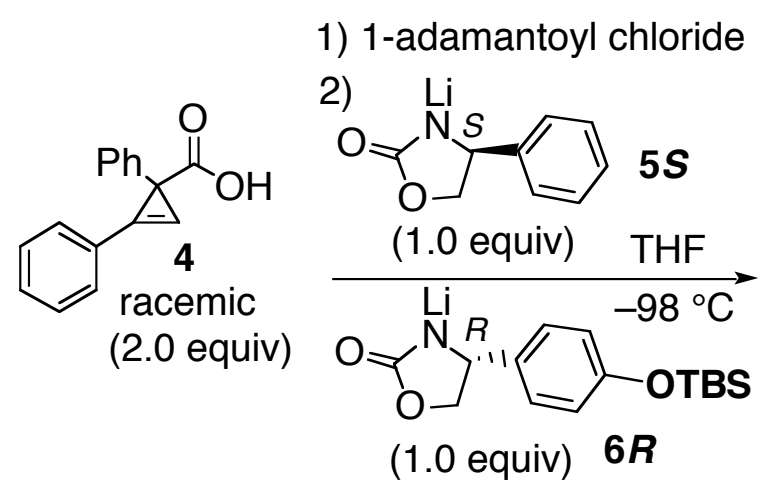

3) TBAF

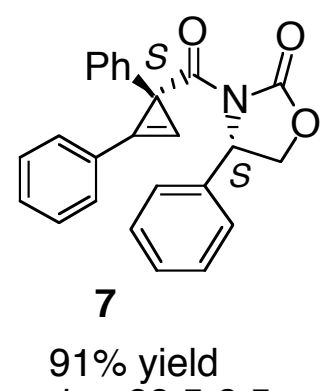

$\mathrm{dr}>$ 99.5:0.5

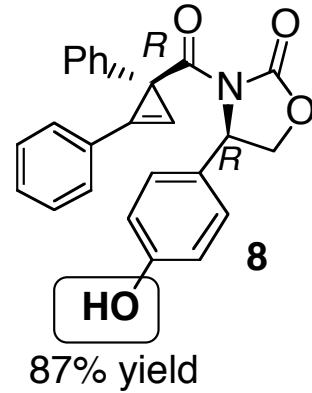

$\mathrm{dr}>99: 1$

The general procedure was followed starting with $142 \mathrm{mg}$ of 1,2-diphenylcycloprop-2ene-1-carboxylic acid (4) ${ }^{1}$ the yield of 7 was $105 \mathrm{mg}(0.28 \mathrm{mmol}, 92 \%$ yield $)$ and the $\mathrm{dr}$ was 99:1. The yield of $\mathbf{8}$ was $106 \mathrm{mg}(0.27 \mathrm{mmol}, 89 \%)$ and the dr was 99:1.

An experiment that started with $5.0 \mathrm{~g}$ of 4 was carried out under similar conditions, except that the mixed anhydride was made using $400 \mathrm{~mL}$ of THF and the reaction was stirred mechanically. The yield of 7 was $3.70 \mathrm{~g}$ (9.71 mmol, 91\% yield) and the $\mathrm{dr}$ was $>99.5: 0.5$. The yield of 8 was $3.63 \mathrm{~g}$ (9.14 mmol, $87 \%$ yield) and the dr was $>99: 1$. The diastereomer purities were determined by HPLC analysis using a CHIRACEL OD column (15\% isopropanol in hexane at $1 \mathrm{~mL} / \mathrm{min})$; minor diastereomers were prepared in separate reactions catalyzed by DMAP. ${ }^{1}$

The absolute configuration of $\mathbf{7}$ had been proven previously by x-ray crystallography.

(4S)-3-[(1S)-1,2-diphenylcycloprop-2-en-1-oyl]-4-phenyloxazolidinone (7): a white solid, $\mathrm{mp}=73-76^{\circ} \mathrm{C}$. $[\alpha]_{\mathrm{D}}-37.7^{\circ}\left(c 1.00, \mathrm{CH}_{2} \mathrm{Cl}_{2}\right) .{ }^{1} \mathrm{H} \mathrm{NMR}\left(\mathrm{CDCl}_{3}, 400 \mathrm{MHz}, \delta\right): 7.60-$ 7.61(m, 2H), 7.40-7.42(m, 3H), 7.29-7.33 (m, 5H), 7.20-7.22 (m, 4H), 7.05-7.07(m,2H), $5.41(\mathrm{dd}, \mathrm{J}=8.9,4.6 \mathrm{~Hz}, 1 \mathrm{H}), 4.62(\operatorname{app~t}, \mathrm{J}=8.9 \mathrm{~Hz}, 1 \mathrm{H}), 4.20(\mathrm{dd}, \mathrm{J}=8.9,4.6 \mathrm{~Hz}, 1 \mathrm{H}) ;{ }^{13} \mathrm{C}$ NMR ( $\left.\mathrm{CDCl}_{3}, 90 \mathrm{MHz}, \delta\right): 173.1(\mathrm{u}), 152.2(\mathrm{u}), 140.4(\mathrm{u}), 138.8(\mathrm{u}), 130.1(\mathrm{dn}), 129.9(\mathrm{dn})$, 129.1(dn), 128.8(dn), 128.6(dn), 128.0(dn), 126.8(dn), 126.6(dn), 126.4(dn), 125.9(u), 122.9(u), 99.8(u), 69.8(u), 57.7(dn), 36.5(u); IR(neat, $\left.\mathrm{cm}^{-1}\right): 3053,2921,1780,1763$, 1595, 1190; HRMS-CI( $\left.\mathrm{NH}_{3}\right) \mathrm{m} / \mathrm{z}$ : [M+], calcd for $\mathrm{C}_{25} \mathrm{H}_{19} \mathrm{NO}_{3}, 381.1365$; found, 381.1375 .

(4R)-3-[(1R)-1,2-diphenylcycloprop-2-en-1-oyl]-4-(4-hydroxyphenyl)oxazolidinone (8) a white semi-solid. $[\alpha]_{\mathrm{D}}+77.6^{\circ}\left(c 1.0, \mathrm{CH}_{2} \mathrm{Cl}_{2}\right) .{ }^{1} \mathrm{H} \mathrm{NMR}\left(\mathrm{CDCl}_{3}, 400 \mathrm{MHz}, \delta\right)$ : 7.58-7.60 (m, 2H), 7.29-7.21 (m, 9H), 7.01-7.03 (m, 2H), 6.84-6.86 (m, 2H), $5.36(\mathrm{dd}$, $\mathrm{J}=4.9,8.9 \mathrm{~Hz}, 1 \mathrm{H}), 5.06(\mathrm{~s}, \mathrm{br}, 1 \mathrm{H}), 4.61(\operatorname{app~t}, \mathrm{J}=8.9 \mathrm{~Hz}, 1 \mathrm{H}), 4.19$ (dd, J=4.7, 8.9Hz, $1 \mathrm{H}) ;{ }^{13} \mathrm{C} \mathrm{NMR}\left(\mathrm{CDCl}_{3}, 90 \mathrm{MHz}, \delta\right): 173.7(\mathrm{u}), 156.4(\mathrm{u}), 152.6(\mathrm{u}), 140.9(\mathrm{u}), 131.3(\mathrm{u})$, 130.5(dn), 130.3(dn), 129.0(dn), 128.8(dn), 128.4(dn), 127.2(dn), 126.8(dn), 126.3(u), 123.2(u), 116.3(dn), 100.1(u), 70.4(u), 57.6(dn), 37.0(u); IR(neat, $\left.\mathrm{cm}^{-1}\right): 3035,2903$, 
1785, 1693, 1595, 1196; HRMS-CI( $\left(\mathrm{NH}_{3}\right) \mathrm{m} / \mathrm{z}:[\mathrm{M}+]$, calcd for $\mathrm{C}_{25} \mathrm{H}_{19} \mathrm{NO}_{4}, 397.1314$; found, 397.1317 .

- Parallel Kinetic Resolution of 2-phenyl-1-( $\alpha$-naphthyl)cycloprop-2-ene-1carboxylic acid (9).<smiles>O=C(O)C1(c2ccccc2)OC1c1cccc2ccccc12</smiles>
1) 1-adamantoyl chloride

2)

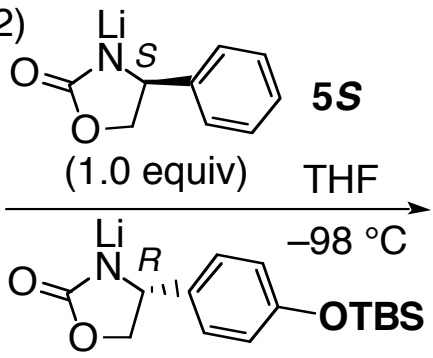

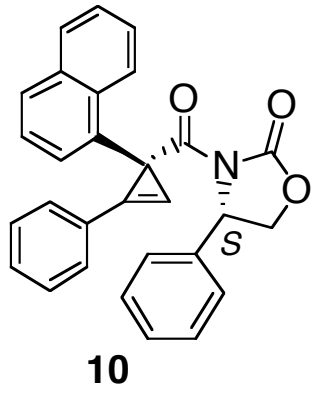

$\mathrm{dr}=97: 3$

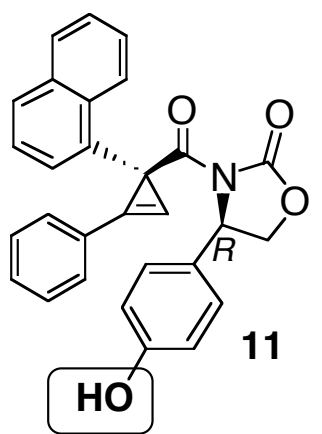

$\mathrm{dr}=97: 3$

(1.0 equiv) $6 \boldsymbol{R}$

3) TBAF

The general procedure for parallel kinetic resolution with $0.60 \mathrm{mmol}$ of 9 gave $106 \mathrm{mg}$ of diasteromerically pure $\mathbf{1 0}(0.27 \mathrm{mmol}, 93 \%$ yield $)$ and $105 \mathrm{mg}$ of diasteromerically pure $11(0.27 \mathrm{mmol}, 90 \%$ yield) . Prior to chromatographic purification, the $\mathrm{dr}$ of $\mathbf{1 0}$ was $97: 3$ and the dr of $\mathbf{1 1}$ was 97:3 (the minor diastereomers were removed by chromatography). These dr's were determined by HPLC analysis using a CHIRACEL OD column (15\% isopropanol in hexane at $1 \mathrm{~mL} / \mathrm{min}$ ); minor diastereomers were prepared in separate reactions catalyzed by DMAP. ${ }^{1}$ An identical experiment gave $\mathbf{1 0}$ in $91 \%$ yield and 98:2 dr, and $\mathbf{1 1}$ in 90\% yield and 97:3 dr. Relative stereochemistry has not been proven for $\mathbf{1 0}$ and $\mathbf{1 1 .}$

(4S)-3-[1-( $\alpha$-Naphthyl)-2-phenylcycloprop-2-en-1-oyl]-4-phenyloxazolidinone (10): a semi-solid, $[\alpha]_{\mathrm{D}}+17.6^{\circ}\left(\mathrm{c} 1.00, \mathrm{CH}_{2} \mathrm{Cl}_{2}\right) .{ }^{1} \mathrm{H}$ NMR $\left(\mathrm{CDCl}_{3}, 400 \mathrm{MHz}, \delta\right): 8.14(\mathrm{~d}, \mathrm{~J}=8.6$ $\mathrm{Hz}, 1 \mathrm{H}), 7.85(\mathrm{~d}, \mathrm{~J}=8.2 \mathrm{~Hz}, 1 \mathrm{H}), 7.71-7.78(\mathrm{~m}, 2 \mathrm{H}), 7.52-7.55(\mathrm{~m}, 2 \mathrm{H}), 7.44(\mathrm{t}, \mathrm{J}=8.0 \mathrm{~Hz}$, $2 \mathrm{H}), 7.30-7.35(\mathrm{~m}, 3 \mathrm{H}), 7.19-7.26(\mathrm{~m}, 5 \mathrm{H}), 7.00-7.03(\mathrm{~m}, 1 \mathrm{H}), 5.2(\mathrm{dd}, \mathrm{J}=6.3,8.7 \mathrm{~Hz}$, 1H), $4.46\left(\right.$ app t, J= 8.8Hz, 1H), $4.00(\mathrm{dd}, \mathrm{J}=6.2,8.8 \mathrm{~Hz}, 1 \mathrm{H}) ;{ }^{13} \mathrm{C} \mathrm{NMR}\left(\mathrm{CDCl}_{3}, 90 \mathrm{MHz}\right.$, ठ): 176.0(u), 152.4(u), 137.7(u), 136.7(u), 133.5(u), 132.6(u), 129.7(2 carbons, dn), 128.9(dn), 128.7(dn), 128.6, (2 carbons, dn), 128.5(dn), 127.8(dn), $126.5(\mathrm{u}), 126.5(\mathrm{dn})$, 126.2(dn), 125.5(dn), 125.2(dn), 124.2(dn), 123.9(u), 102.8(u), 69.9(u), 58.7(dn), 37.3(u); IR( neat, $\mathrm{cm}^{-1}$ ): 3036, 2956, 1783, 1750, 1596, 1450, 1190; HRMS-CI $\left(\mathrm{NH}_{3}\right) \mathrm{m} / \mathrm{z}$ : [M+Na], calcd for $\mathrm{C}_{29} \mathrm{H}_{21} \mathrm{NO}_{3}, 454.1419$; found, 454.1436.

(4R)-3-[(1-( $\alpha$-Naphthyl)-2-phenylcycloprop-2-en-1-oyl]-4-(4-hydroxyphenyl)

oxazolidinone (11): a semi-solid, $[\alpha]_{\mathrm{D}}+53.8^{\circ}\left(\mathrm{c} 1.03, \mathrm{CH}_{2} \mathrm{Cl}_{2}\right) .{ }^{1} \mathrm{H}$ NMR $\left(\mathrm{CDCl}_{3}\right.$, $400 \mathrm{MHz}, \delta): 8.11(\mathrm{~d}, \mathrm{~J}=8.5 \mathrm{~Hz}, 1 \mathrm{H}), 7.84(\mathrm{~d}, \mathrm{~J}=8.2 \mathrm{~Hz}, 1 \mathrm{H}), 7.71-7.77(\mathrm{~m}, 3 \mathrm{H}), 7.51-$ 
$7.54(\mathrm{~m}, 2 \mathrm{H}), 7.42-7.46(\mathrm{~m}, 3 \mathrm{H}), 7.26-7.34(\mathrm{~m}, 2 \mathrm{H}), 6.86-6.89(\mathrm{~m}, 2 \mathrm{H}), 6.60-6.62(\mathrm{~m}$, 2H), $5.26(\mathrm{dd}, \mathrm{J}=6.2,8.6 \mathrm{~Hz}, 1 \mathrm{H}), 5.16(\mathrm{~s}, 1 \mathrm{H}), 4.43($ app t, J=8.8 Hz, 1H), 3.98 (dd, $\mathrm{J}=6.2 \mathrm{~Hz}, 8.6 \mathrm{~Hz}, 1 \mathrm{H}) ;{ }^{13} \mathrm{C} \mathrm{NMR}\left(\mathrm{CDCl}_{3}, 90 \mathrm{MHz}, \delta\right): 176.2(\mathrm{u}), 155.8(\mathrm{u}), 152.5(\mathrm{u})$, $136.6(\mathrm{u}), 133.5$ (2 carbons, u), $132.6(\mathrm{u}), 129.79(\mathrm{dn}), 129.75(\mathrm{dn}), 129.73(\mathrm{dn}), 128.7$ (dn), $128.6(\mathrm{dn}), 128.2(\mathrm{dn}), 127.8(\mathrm{dn}), 126.5(\mathrm{u}), 126.2(\mathrm{dn}), 125.5(\mathrm{dn}), 125.2(\mathrm{dn})$, $124.1(\mathrm{dn}), 123.9(\mathrm{u}), 115.6(\mathrm{dn}), 102.7(\mathrm{u}), 70.0(\mathrm{u}), 58.2(\mathrm{dn}), 37.4(\mathrm{u})$; IR(neat, $\left.\mathrm{cm}^{-1}\right)$ : 3035, 2903, 1785, 1693, 1595, 1196; HRMS-CI(NH$) \mathrm{m} / \mathrm{z}$ : [M+Na], calcd for $\mathrm{C}_{29} \mathrm{H}_{21}$ $\mathrm{NO}_{4}, 470.1368$; found, 470.1356 .

\section{- Parallel Kinetic Resolution of 2-phenylcycloprop-2-ene-1,1-dioic acid monoethyl ester (12).}

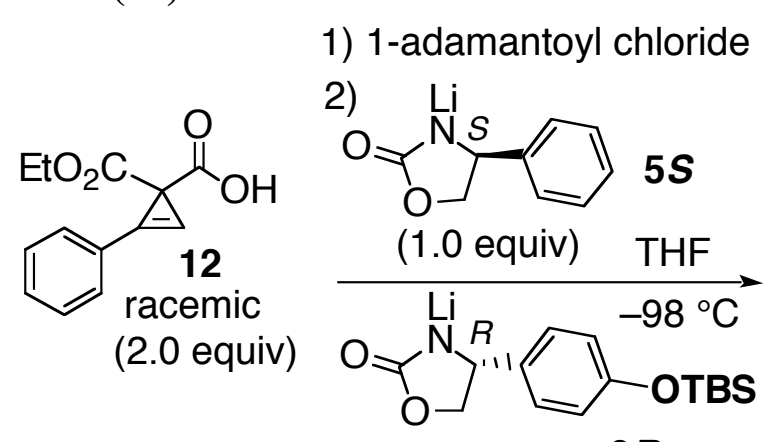

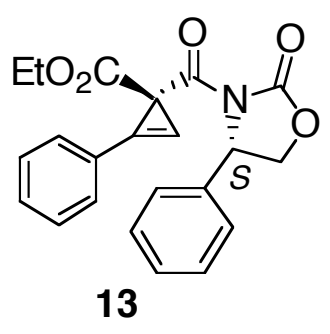

$\mathrm{dr}=98: 2$

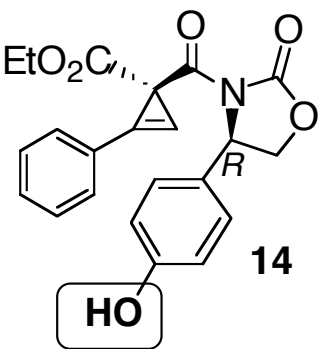

$\mathrm{dr}=97: 3$

(1.0 equiv) $6 R$

\section{3) TBAF}

The general procedure for parallel kinetic resolution with $0.60 \mathrm{mmol}$ of $\mathbf{1 2}$ gave $103 \mathrm{mg}$ of diasteromerically pure $13(0.27 \mathrm{mmol}, 91 \%$ yield $)$ and $105 \mathrm{mg}$ of diasteromerically pure 14 (0.27 mmol, 89\% yield) . Prior to chromatographic purification, the $\mathrm{dr}$ of 12 was 98:2 and the dr of $\mathbf{1 4}$ was 97:3 (the minor diastereomers were removed by chromatography). These dr's were determined by HPLC analysis using a CHIRACEL OD column (15\% isopropanol in hexane at $1 \mathrm{~mL} / \mathrm{min})$; minor diastereomers were prepared in separate reactions catalyzed by DMAP. ${ }^{1}$ An identical experiment gave $\mathbf{1 3}$ in 90\% yield and 98:2 dr, and 14 in 88\% yield and 98:2 dr. Relative stereochemistry has not been proven for $\mathbf{1 3}$ and $\mathbf{1 4}$.

(4S)-4-Phenyl-3-(2-phenyl-1-ethoxycarbonyl-cycloprop-2-en-1-oyl)oxazolidinone (13): a semi-solid, $[\alpha]_{\mathrm{D}}+138.3^{\circ}$ (c 1.03, $\left.\mathrm{CH}_{2} \mathrm{Cl}_{2}\right)$. ${ }^{1} \mathrm{H}$ NMR $\left(\mathrm{CDCl}_{3}, 400 \mathrm{MHz}, \delta\right)$ : 7.747.77(m, 2H), 7.38-7.42(m, 8H), 6.95(s, 1H), 5.46(dd, J 4.5, 8.9 Hz, 1H), 4.77 (app t, $\mathrm{J}=8.9 \mathrm{~Hz}, 1 \mathrm{H}), 4.31(\mathrm{dd}, \mathrm{J}=4.5,8.9 \mathrm{~Hz}, 1 \mathrm{H}), 4.21-4.24(\mathrm{~m}, 2 \mathrm{H}), 1.25-1.29(\mathrm{t}, \mathrm{J}=7.4 \mathrm{~Hz}$, $3 \mathrm{H}) ;{ }^{13} \mathrm{C} \mathrm{NMR}\left(\mathrm{CDCl}_{3}, 90 \mathrm{MHz}, \delta\right): 171.1(\mathrm{u}), 170.0(\mathrm{u}), 153.9(\mathrm{u}), 138.6(\mathrm{u}), 130.5(\mathrm{dn})$, 130.4(dn), 129.1(dn), 128.7(dn), 128.6(dn), 125.9(dn), 124.4(u), 113.3(u), 96.2(u), 70.5(u), 61.3(u), 57.9(dn), 38.6(u), 14.2(dn); IR (neat, $\left.\mathrm{cm}^{-1}\right): 3056,2928,1780,1763$, 1595, 1190; HRMS-CI $\left(\mathrm{NH}_{3}\right) \mathrm{m} / \mathrm{z}$ : [M+Na], calcd for $\mathrm{C}_{22} \mathrm{H}_{19} \mathrm{NO}_{5}, 400.1161$; found, 400.1174 .

(4R)-4-(4-Hydroxyphenyl)-3-(2-phenyl-1-ethoxycarbonyl-cycloprop-2-en-1oyl)oxazolidinone (14): a semi-solid, $[\alpha]_{\mathrm{D}}-12.6^{\circ}$ (c 1.03, THF). ${ }^{1} \mathrm{H} \mathrm{NMR}\left(\mathrm{CDCl}_{3}\right.$, 


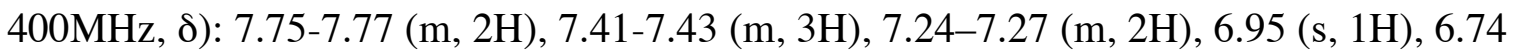
(d, J=8.5 Hz, 2H), 5.94 (br s., 1H), 5.39 (dd, J= 4.8, $8.9 \mathrm{~Hz}, 1 \mathrm{H}), 4.72$ (t, J=8.9Hz, $1 \mathrm{H})$, $4.30(\mathrm{dd}, \mathrm{J}=4.8,8.9 \mathrm{~Hz}, 1 \mathrm{H}), 4.16-4.26(\mathrm{~m}, 2 \mathrm{H}), 1.24-1.28(\mathrm{t}, \mathrm{J}=7.1 \mathrm{~Hz}, 3 \mathrm{H}) ;{ }^{13} \mathrm{C} \mathrm{NMR}$ $\left(\mathrm{CDCl}_{3}, 100 \mathrm{MHz}, \delta\right): 171.4(\mathrm{u}), 170.3(\mathrm{u}), 156.2(\mathrm{u}), 153.9(\mathrm{u}), 130.5(\mathrm{dn}), 130.4(\mathrm{dn})$, 130.2(u), 128.7(dn), 127.6(dn), 124.3(u), 115.9(dn), 113.2(u), 96.1(u), 70.6(u), 61.5(u), 57.6(dn), 36.6(u), 14.2(dn); IR(neat, $\left.\mathrm{cm}^{-1}\right): 3037,2908,1781,1760,1595,1185$; HRMS$\mathrm{CI}\left(\mathrm{NH}_{3}\right) \mathrm{m} / \mathrm{z}$ : [M+Na], calcd for $\mathrm{C}_{22} \mathrm{H}_{19} \mathrm{NO}_{6}$, 416.1110; found, 416.1123.

\section{- Parallel Kinetic Resolution of 2-butylcycloprop-2-ene-1,1-dioic acid monoethyl ester (15)}
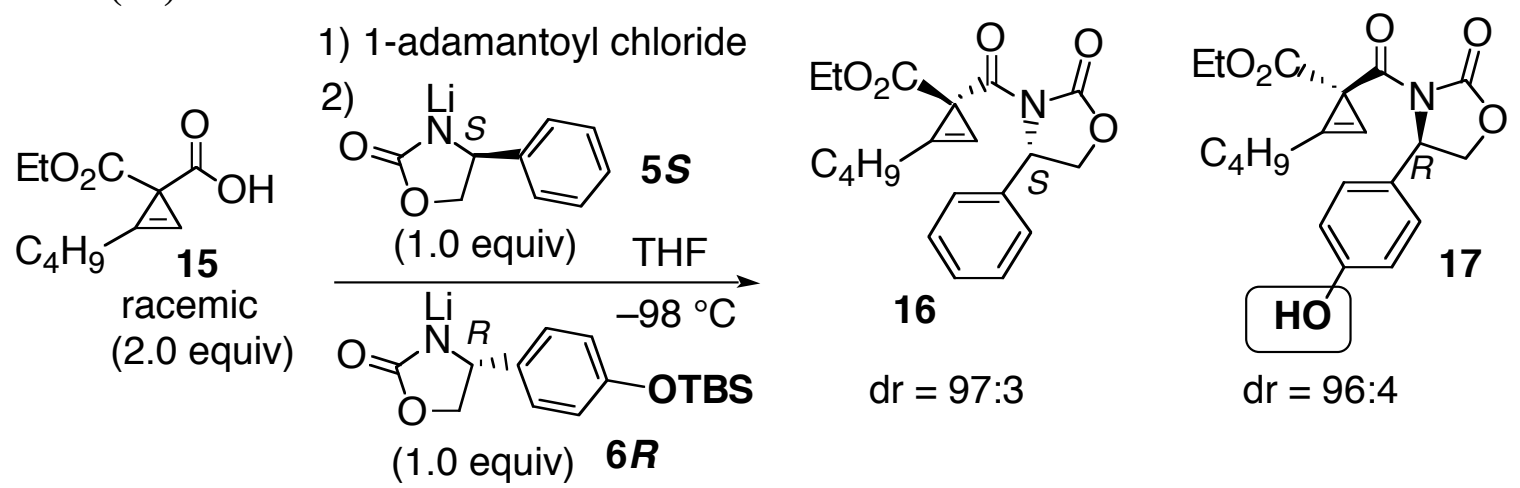

3) TBAF

The general procedure for parallel kinetic resolution with $0.60 \mathrm{mmol}$ of $\mathbf{1 5}$ gave $95 \mathrm{mg}$ of diasteromerically pure $16(0.27 \mathrm{mmol}, 91 \%$ yield $)$ and $97 \mathrm{mg}$ of diasteromerically pure 17 (0.27 mmol, 89\% yield). Prior to chromatographic purification, the dr of $\mathbf{1 6}$ was 97:3 and the dr of 17 was 96:4 (the minor diastereomers were removed by chromatography). These dr's were determined by HPLC analysis using a CHIRALCEL OD column (10\% isopropanol in hexane at $1 \mathrm{~mL} / \mathrm{min}$ ); minor diastereomers were prepared in separate reactions catalyzed by DMAP. ${ }^{1}$ An identical experiment gave 16 in 90\% yield and 98:2 dr, and $\mathbf{1 7}$ in 87\% yield and 96:4 dr. Relative stereochemistry has not been proven for $\mathbf{1 6}$ and 17.

(4S)-4-Phenyl-3-(2-butyl-1-ethoxycarbonyl-cycloprop-2-en-1-oyl)oxazolidinone

(16): a semi-solid, $[\alpha]_{\mathrm{D}}+48.6^{\circ}\left(c 1.0, \mathrm{CH}_{2} \mathrm{Cl}_{2}\right) .{ }^{1} \mathrm{H} \mathrm{NMR}\left(\mathrm{CDCl}_{3}, 400 \mathrm{MHz}, \delta\right): 7.39-7.40$ $(\mathrm{m}, 5 \mathrm{H}), 6.44(\mathrm{t}, \mathrm{J}=1.4 \mathrm{~Hz}, 1 \mathrm{H}), 5.43(\mathrm{dd}, \mathrm{J}=4.2,8.8 \mathrm{~Hz}, 1 \mathrm{H}), 4.73(\operatorname{app~t}, \mathrm{J}=8.8 \mathrm{~Hz}, 1 \mathrm{H})$, $4.29(\mathrm{dd}, \mathrm{J}=4.2,8.9 \mathrm{~Hz}, 1 \mathrm{H}), 4.17-4.21(\mathrm{~m}, 2 \mathrm{H}), 2.58-2.59(\mathrm{~m}, 2 \mathrm{H}), 1.55-1.62(\mathrm{~m}, 2 \mathrm{H})$, $1.34-1.38(\mathrm{~m}, 2 \mathrm{H}), 1.25-1.29(\mathrm{t}, \mathrm{J}=7.1 \mathrm{~Hz}, 3 \mathrm{H}), 0.86-0.90(\mathrm{t}, \mathrm{J}=7.4 \mathrm{~Hz}, 3 \mathrm{H}) ;{ }^{13} \mathrm{C}$ $\mathrm{NMR}\left(\mathrm{CDCl}_{3}, 90 \mathrm{MHz}, \delta\right): 171.8(\mathrm{u}), 170.9(\mathrm{u}), 153.6(\mathrm{u}), 138.8(\mathrm{u}), 129.1(\mathrm{dn}), 128.6(\mathrm{dn})$, 125.9(dn), 116.6(u), 95.0(u), 70.3(u), 61.1(u), 57.6(dn), 35.1(u), 28.6(u), 24.0(u), 22.1(u), 14.2(dn), 13.6(dn); IR(neat, $\left.\mathrm{cm}^{-1}\right): 3056,2928,1780,1763,1595,1190 ;$ HRMS-CI(NH $\left.\mathrm{NH}_{3}\right)$ $\mathrm{m} / \mathrm{z}$ : [M+Na], calcd for $\mathrm{C}_{20} \mathrm{H}_{23} \mathrm{NO}_{5}, 380.1474$; found, 380.1484 .

(4R)-4-(4-Hydroxyphenyl)-3-(2-butyl-1-ethoxycarbonyl-cycloprop-2-en-1oyl)oxazolidinone (17): a semi-solid, $[\alpha]_{\mathrm{D}}+43.8^{\circ}\left(c\right.$ 1.03, $\left.\mathrm{CH}_{2} \mathrm{Cl}_{2}\right) .{ }^{1} \mathrm{H}$ NMR $\left(\mathrm{CDCl}_{3}\right.$, $400 \mathrm{MHz}, \delta): 7.27-7.30(\mathrm{~m}, 2 \mathrm{H}), 6.79-6.82(\mathrm{~m}, 2 \mathrm{H}), 6.44(\mathrm{t}, \mathrm{J}=1.4 \mathrm{~Hz}, 1 \mathrm{H}), 5.39$ (dd, J= 
$5.1,8.9 \mathrm{~Hz}, 1 \mathrm{H}), 5.23$ (br s, 1H), 4.69-4.73 (app t, J=8.9Hz, 1H), 4.26-4.29 (dd, J= 5.1, $8.9 \mathrm{~Hz}, 1 \mathrm{H}), 4.15-4.19(\mathrm{~m}, 2 \mathrm{H}), 2.51-2.62(\mathrm{~m}, 2 \mathrm{H}), 1.57-1.61(\mathrm{~m}, 2 \mathrm{H}), 1.38-1.41(\mathrm{~m}, 2 \mathrm{H})$, 1.24-1.28 (t, J=7.1Hz, 3H), 0.89-0.93 (t, J=7.4Hz, 3H); ${ }^{13} \mathrm{C} \mathrm{NMR}\left(\mathrm{CDCl}_{3}, 90 \mathrm{MHz}, \delta\right)$ : 171.9(u), 171.2(u), 155.9(u), 153.7(u), 130.6(u), 127.8(dn), 116.4(u), 115.9(dn), 94.4(u), 70.5(u), 61.1(u), 57.4(dn), 35.6(u), 28.6(u), 24.0(u), 22.1(u), 14.2(dn), 13.7(dn); IR(neat, $\left.\mathrm{cm}^{-1}\right): 3037,2908,1781,1760,1595,1185 ; \mathrm{HRMS}-\mathrm{CI}\left(\mathrm{NH}_{3}\right) \mathrm{m} / \mathrm{z}:[\mathrm{M}+]$, calcd for $\mathrm{C}_{20} \mathrm{H}_{23}$ $\mathrm{NO}_{6}, 373.1525$; found, 373.1519 .

\section{- Parallel Kinetic Resolution of 2-phenyl-1-(2-thienyl)cycloprop-2-ene-1-carboxylic acid (18).}
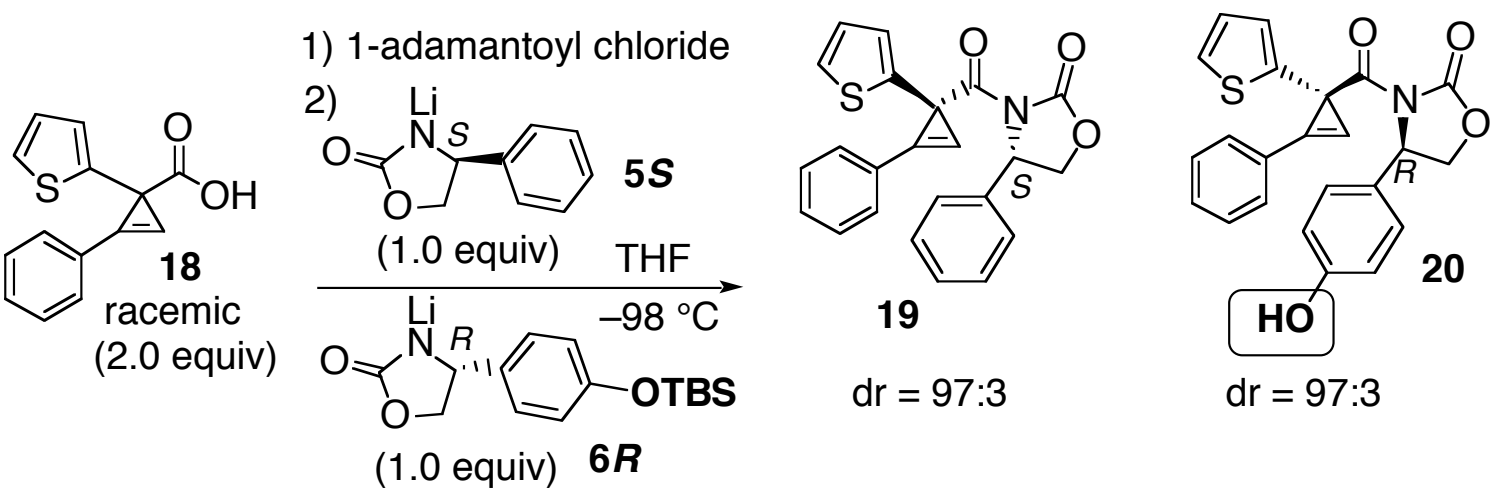

3) TBAF

The general procedure for parallel kinetic resolution with $0.60 \mathrm{mmol}$ of 18 gave $106 \mathrm{mg}$ of diasteromerically pure $19(0.27 \mathrm{mmol}, 91 \%$ yield $)$ and $105 \mathrm{mg}$ of diasteromerically pure 20 ( $0.26 \mathrm{mmol}, 87 \%$ yield). Prior to chromatographic purification, the $\mathrm{dr}$ of $\mathbf{1 9}$ was 97:3 and the dr of $\mathbf{2 0}$ was 97:3 (the minor diastereomers were removed by chromatography). These dr's were determined by HPLC analysis using a CHIRACEL OD column ( $15 \%$ isopropanol in hexane at $1 \mathrm{~mL} / \mathrm{min})$; minor diastereomers were prepared in separate reactions catalyzed by DMAP. ${ }^{1}$ An identical experiment gave 19 in 90\% yield and 98:2 dr, and $\mathbf{2 0}$ in $87 \%$ yield and 97:3 dr. Relative stereochemistry has not been proven for $\mathbf{1 9}$ and $\mathbf{2 0 .}$

(4S)-3-[1-(2-thienyl)-2-phenylcycloprop-2-en-1-oyl]-4-phenyloxazolidinone (19): a semi-solid, $[\alpha]_{\mathrm{D}}+78.6^{\circ}\left(\mathrm{c} 1.0, \mathrm{CH}_{2} \mathrm{Cl}_{2}\right) .{ }^{1} \mathrm{H} \mathrm{NMR}\left(\mathrm{CDCl}_{3}, 400 \mathrm{MHz}, \delta\right): 7.68-7.71(\mathrm{~m}$, $2 \mathrm{H}), 7.34-7.43(\mathrm{~m}, 8 \mathrm{H}), 7.18(\mathrm{~s}, 1 \mathrm{H}), 7.10(\mathrm{dd}, \mathrm{J}=1.1,5.1 \mathrm{~Hz}, 1 \mathrm{H}), 6.88(\mathrm{dd}, \mathrm{J}=3.6,5.1$ $\mathrm{Hz}, 1 \mathrm{H}), 6.74(\mathrm{dd}, \mathrm{J}=1.2,3.5 \mathrm{~Hz}), 5.41(\mathrm{dd}, \mathrm{J}=4.7,8.8 \mathrm{~Hz}, 1 \mathrm{H}), 4.69(\operatorname{app~t}, \mathrm{J}=8.9 \mathrm{~Hz}, 1 \mathrm{H})$, $4.26(\mathrm{dd}, \mathrm{J}=4.7,8.9 \mathrm{~Hz}, 1 \mathrm{H}) ;{ }^{13} \mathrm{C} \mathrm{NMR}\left(\mathrm{CDCl}_{3}, 90 \mathrm{MHz}, \delta\right): 171.7(\mathrm{u}), 152.3(\mathrm{u}), 146.3(\mathrm{u})$, 138.5(u), 130.2(dn), 130.1(dn), 129.1(dn), 128.8(dn), 128.7(dn), 126.8(dn), 126.4(dn), 125.3(u), 124.5(dn), 123.7(dn), 123.5(u), 100.8(u), 70.0(u), 57.9(dn), 32.7(u); IR( neat, $\left.\mathrm{cm}^{-1}\right): 3033,2956,1783,1763,1605,1450,1190 ; \mathrm{HRMS}-\mathrm{CI}\left(\mathrm{NH}_{3}\right) \mathrm{m} / \mathrm{z}:[\mathrm{M}+\mathrm{Na}]$, calcd for $\mathrm{C}_{23} \mathrm{H}_{17} \mathrm{NO}_{3} \mathrm{~S}, 410.0827$; found, 410.0841 . 
(4R)-3-[1-(2-thienyl)-2-phenylcycloprop-2-en-1-oyl]-4-(4-hydroxyphenyl)

oxazolidinone (20): a semi-solid, $[\alpha]_{\mathrm{D}}-36.3^{\circ} \quad\left(\mathrm{c} 1.05, \mathrm{CH}_{2} \mathrm{Cl}_{2}\right) .{ }^{1} \mathrm{H}$ NMR $\left(\mathrm{CDCl}_{3}\right.$, $400 \mathrm{MHz}, \delta): 7.68-7.70(\mathrm{~m}, 2 \mathrm{H}), 7.35-7.37(\mathrm{~m}, 3 \mathrm{H}), 7.21-7.24(\mathrm{~m}, 2 \mathrm{H}), 7.17(\mathrm{~s}, 1 \mathrm{H})$, 7.09-7.10 (dd, J=1.3Hz, 5.1Hz, 1H), 6.84-6.89 (m, 3H), 6.72-6.73 (dd, J = 0.9, 3.4Hz, $1 \mathrm{H}), 5.36(\mathrm{dd}, \mathrm{J}=4.7,8.7 \mathrm{~Hz}, 1 \mathrm{H}), 4.66(\operatorname{app~t}, \mathrm{J}=8.8 \mathrm{~Hz}, 1 \mathrm{H}), 4.25(\mathrm{dd}, \mathrm{J}=4.7,8.7 \mathrm{~Hz}$, $1 \mathrm{H}) ;{ }^{13} \mathrm{C}$ NMR $\left(\mathrm{CDCl}_{3}, 90 \mathrm{MHz}, \delta\right): 171.9(\mathrm{u}), 156.0(\mathrm{u}), 152.4(\mathrm{u}), 146.4(\mathrm{u}), 130.6(\mathrm{u})$, 130.2(dn), 130.1(dn), 128.7(dn), 128.1(dn), 126.8(dn), 125.3(u), 124.4(dn), 123.7(dn), 123.3(u), 115.9(dn), 100.7(u), 70.1(u), 57.4(dn), 32.8(u); IR( neat, $\left.\mathrm{cm}^{-1}\right): 3035,2903$, 1785, 1693, 1595, 1196; HRMS-CI $\left(\mathrm{NH}_{3}\right) \mathrm{m} / \mathrm{z}$ : [M+Na], calcd for $\mathrm{C}_{23} \mathrm{H}_{17} \mathrm{NO}_{4} \mathrm{~S}, 426.0776$; found, 426.0789 .

- Parallel Kinetic Resolution of 2-phenyl-1-(methoxymethyl)cycloprop-2-ene-1carboxylic acid (21).

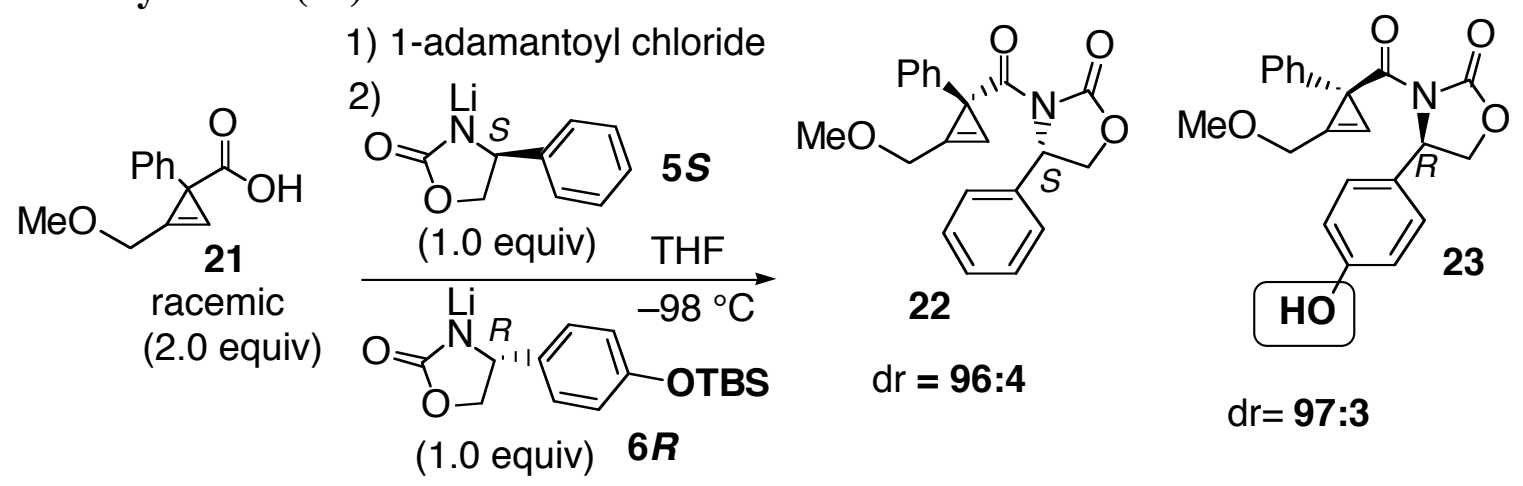

3) TBAF

The general procedure for parallel kinetic resolution with $0.60 \mathrm{mmol}$ of $\mathbf{2 1}$ gave $95 \mathrm{mg}$ of diasteromerically pure $\mathbf{2 2}(0.28 \mathrm{mmol}, 93 \%$ yield $)$ and $97 \mathrm{mg}$ of diasteromerically pure 23 (0.27 mmol, 89\% yield). Prior to chromatographic purification, the dr of 22 was $96: 4$ and the dr of $\mathbf{2 3}$ was 97:3 (the minor diastereomers were removed by chromatography). These dr's were determined by HPLC analysis using a CHIRACEL OD column ((15 isopropanol in hexane at $1 \mathrm{~mL} / \mathrm{min}$ ); minor diastereomers were prepared in separate reactions catalyzed by DMAP. ${ }^{1}$ An identical experiment gave $\mathbf{2 2}$ in $90 \%$ yield and 97:3 dr, and $\mathbf{2 3}$ in $87 \%$ yield and 97:3 dr. Relative stereochemistry has not been proven for $\mathbf{2 2}$ and 23.

(4S)-3-[1-phenyl-2-(methoxymethyl)cycloprop-2-en-1-oyl]-4-phenyloxazolidinone (22): a white solid, $\mathrm{mp}=56-59{ }^{\circ} \mathrm{C}$. $[\alpha]_{\mathrm{D}}+163.5^{\circ}$ (c 1.00, THF). ${ }^{1} \mathrm{H}$ NMR $\left(\mathrm{CDCl}_{3}\right.$, $400 \mathrm{MHz}, \delta): 7.40-7.42(\mathrm{~m}, 3 \mathrm{H}), 7.32-7.33(\mathrm{~m}, 2 \mathrm{H}), 7.23-7.27(\mathrm{~m}, 3 \mathrm{H}), 7.16(\mathrm{~m}, 1 \mathrm{H})$, 7.01-7.04 (m,2H), $5.46(\mathrm{dd}, \mathrm{J}=4.8,8.8 \mathrm{~Hz}, 1 \mathrm{H}), 4.50-4.68(\mathrm{~m}, 3 \mathrm{H}), 4.25(\mathrm{dd}, \mathrm{J}=4.8,8.9$ $\mathrm{Hz}, 1 \mathrm{H}), 3.42(\mathrm{~s}, 3 \mathrm{H}) ;{ }^{13} \mathrm{C}$ NMR $\left(\mathrm{CDCl}_{3}, 90 \mathrm{MHz}, \delta\right): 173.5(\mathrm{u}), 152.2(\mathrm{u}), 141.3(\mathrm{u})$, 138.4(u), 129.1(dn), 128.9(dn), 128.1 (dn), 126.6(dn), 126.5(dn), 126.4(dn), 119.1(u), 104.7(u), 69.8(u), 66.5(u), 58.7(dn), 57.7(dn), 36.8(u); IR(neat, $\left.\mathrm{cm}^{-1}\right)$ : 3053, 2921, 1780, 1763, 1595, 1190; HRMS-CI $\left(\mathrm{NH}_{3}\right) \mathrm{m} / \mathrm{z}$ : [M+], calcd for $\mathrm{C}_{21} \mathrm{H}_{19} \mathrm{NO}_{4}, 349.1314$; found, 349.1313 .

(4R)-3-[1-phenyl-2-(methoxymethyl)cycloprop-2-en-1-oyl]-4-(4hydroxy)phenyloxazolidinone (23): a white semi-solid, $[\alpha]_{D}+43.8^{\circ}$ (1.03, THF). ${ }^{1} \mathrm{H}$ 
NMR $\left(\mathrm{CDCl}_{3}, 400 \mathrm{MHz}, \delta\right): 7.21-7.27(\mathrm{~m}, 3 \mathrm{H}), 7.16-7.18(\mathrm{~m}, 2 \mathrm{H}), 7.05-7.06$ (app t, $\mathrm{J}=1.6 \mathrm{~Hz}, 1 \mathrm{H}), 6.95-6.98(\mathrm{~m}, 2 \mathrm{H}), 6.80-6.82(\mathrm{~m}, 2 \mathrm{H}), 5.86(\mathrm{~s}, 1 \mathrm{H}), 5.35-5.38(\mathrm{q}, \mathrm{J}=5.0$, $8.8 \mathrm{~Hz}, 1 \mathrm{H}), 4.61-4.68(\mathrm{~m}, 2 \mathrm{H}), 4.40-4.44(\mathrm{dd}, \mathrm{J}=1.6,16.6 \mathrm{~Hz}, 1 \mathrm{H}), 4.20(\mathrm{dd}, \mathrm{J}=5.0$, $8.9 \mathrm{~Hz}, 1 \mathrm{H}), 3.36(\mathrm{~s}, 3 \mathrm{H}) ;{ }^{13} \mathrm{C} \mathrm{NMR}\left(\mathrm{CDCl}_{3}, 90 \mathrm{MHz}, \delta\right): 174.0(\mathrm{u}), 156.4(\mathrm{u}), 152.3(\mathrm{u})$, 140.4(u), 130.1(u), 128.2(dn), 128.1(dn), 126.6(2 carbons, dn), 121.6(u), 115.9(dn), 101.9(u), 70.0(u), 66.2(u), 58.6(dn), 57.3(dn), 36.9(u); IR(neat, $\left.\mathrm{cm}^{-1}\right): 3037,2906,1787$, 1760, 1595, 1195; HRMS-CI $\left(\mathrm{NH}_{3}\right) \mathrm{m} / \mathrm{z}$ : [M+], calcd for $\mathrm{C}_{26} \mathrm{H}_{19} \mathrm{NO}_{3}$, 393.1365; found, 393.1352 .

Parallel Kinetic Resolution of 2-butyl-1-phenylcycloprop-2-ene-1-carboxylic acid (24).

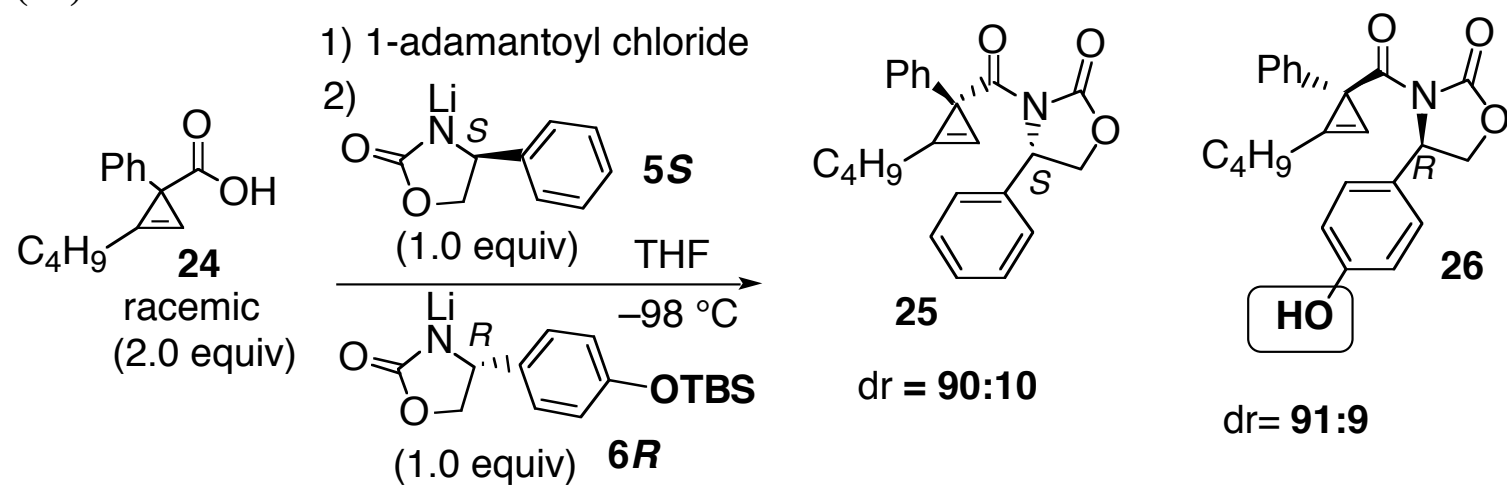

3) TBAF

The general procedure for parallel kinetic resolution with $0.60 \mathrm{mmol}$ of $\mathbf{2 4}$ gave $94 \mathrm{mg}$ of diasteromerically pure 25 ( $0.26 \mathrm{mmol}, 87 \%$ yield) and $94 \mathrm{mg}$ of diasteromerically pure 26 (0.25 mmol, 83\% yield) . Prior to chromatographic purification, the $\mathrm{dr}$ of $\mathbf{2 5}$ was 90:10 and the dr of $\mathbf{2 6}$ was 91:9 (the minor diastereomers were removed by chromatography). These dr's were determined by HPLC analysis using a CHIRACEL OD column (10\% isopropanol in hexane at $1 \mathrm{~mL} / \mathrm{min})$; minor diastereomers were prepared in separate reactions catalyzed by DMAP. ${ }^{1}$ An identical experiment gave 25 in $88 \%$ yield and $91: 9 \mathrm{dr}$, and $\mathbf{2 6}$ in $85 \%$ yield and 90:10 dr. Relative stereochemistry has not been proven for $\mathbf{2 5}$ and $\mathbf{2 6}$.

(4S)-3-(1-phenyl-2-butylcycloprop-2-en-1-oyl)-4-phenyloxazolidinone (25): a semisolid, $[\alpha]_{\mathrm{D}}+28.6^{\circ}$ (c 0.95, $\left.\mathrm{CH}_{2} \mathrm{Cl}_{2}\right) .{ }^{1} \mathrm{H}$ NMR $\left(\mathrm{CDCl}_{3}, 400 \mathrm{MHz}, \delta\right): 7.40-7.45(\mathrm{~m}, 3 \mathrm{H})$, 7.31-7.33(m, 2H), 7.19-7.25(m, 3H), 6.94-6.97(m, 2H), $6.69(\mathrm{t}, \mathrm{J}=1.3 \mathrm{~Hz}, 1 \mathrm{H}), 5.40$ (dd, $\mathrm{J}=5.0,8.9 \mathrm{~Hz}, 1 \mathrm{H}), 4.63(\operatorname{app~t}, \mathrm{J}=9.0 \mathrm{~Hz}, 1 \mathrm{H}), 4.20(\mathrm{dd}, \mathrm{J}=5.0,8.9 \mathrm{~Hz}, 1 \mathrm{H}), 2.48-2.63(\mathrm{~m}$, $2 \mathrm{H}), 1.46-1.57(\mathrm{~m}, 2 \mathrm{H}), 1.23-1.30(\mathrm{~m}, 2 \mathrm{H}), 0.81(\mathrm{t}, \mathrm{J}=7.3 \mathrm{~Hz}, 3 \mathrm{H}) ;{ }^{13} \mathrm{C} \mathrm{NMR}\left(\mathrm{CDCl}_{3}\right.$,

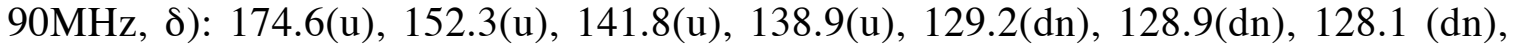
126.8(dn), 126.7(dn), 126.2(dn), 125.5(u), 98.9(u), 69.9(u), 57.9(dn), 35.9(u), 29.1(u), 24.5(u), 22.4(u), 13.8(dn); IR( neat, $\left.\mathrm{cm}^{-1}\right)$ : 3053, 2921, 1780, 1763, 1595, 1190; HRMS$\mathrm{CI}\left(\mathrm{NH}_{3}\right) \mathrm{m} / \mathrm{z}$ : $[\mathrm{M}+\mathrm{H}]$, calcd for $\mathrm{C}_{23} \mathrm{H}_{23} \mathrm{NO}_{3}, 362.1756$; found, 362.1747.

(4S)-3-(1-phenyl-2-butylcycloprop-2-en-1-oyl)-4-(4-hydroxyphenyl)oxazolidinone

(26): a semi-solid, $[\alpha]_{\mathrm{D}}+6.7^{\circ}\left(\mathrm{c} 0.93, \mathrm{CH}_{2} \mathrm{Cl}_{2}\right) .{ }^{1} \mathrm{H} \mathrm{NMR}\left(\mathrm{CDCl}_{3}, 400 \mathrm{MHz}, \delta\right): 7.23-7.27$ 
$(\mathrm{m}, 2 \mathrm{H}), 7.15-7.22(\mathrm{~m}, 3 \mathrm{H}), 7.00-7.03(\mathrm{~m}, 2 \mathrm{H}), 6.82-6.83(\mathrm{t}, \mathrm{J}=1.6 \mathrm{~Hz}, 1 \mathrm{H}), 6.78-6.82(\mathrm{~m}$, $2 \mathrm{H}), 5.58(\mathrm{~s}, 1 \mathrm{H}), 5.41(\mathrm{dd}, \mathrm{J}=5.0,8.8 \mathrm{~Hz}, 1 \mathrm{H}), 4.62(\operatorname{app~t}, \mathrm{J}=8.9 \mathrm{~Hz}, 1 \mathrm{H}), 4.21(\mathrm{dd}, \mathrm{J}=$ 5.1. $8.9 \mathrm{~Hz}, 1 \mathrm{H}), 2.61-2.65(\mathrm{~m}, 1 \mathrm{H}), 2.51-2.58(\mathrm{~m}, 1 \mathrm{H}), 1.51-1.59(\mathrm{~m}, 2 \mathrm{H}), 1.26-1.39(\mathrm{~m}$, $2 \mathrm{H}), 0.86-0.89(\mathrm{t}, \mathrm{J}=7.3 \mathrm{~Hz}, 3 \mathrm{H}) ;{ }^{13} \mathrm{C} \mathrm{NMR}\left(\mathrm{CDCl}_{3}, 100 \mathrm{MHz}, \delta\right): 175.2(\mathrm{u}), 156.1(\mathrm{u})$, 152.2(u), 142.4(u), 130.3(u), 128.2(dn), 128.0(dn), 126.6(dn), 126.1(dn), 122.5(u), 115.8(dn), 101.4(u), 69.9(u), 57.4(dn), 36.1(u), 29.0(u), 24.6(u), 22.2(u), 13.7(dn); IR(neat, $\left.\mathrm{cm}^{-1}\right): 3037,2906,1783,1760,1605,1095 ; \mathrm{HRMS}-\mathrm{CI}\left(\mathrm{NH}_{3}\right) \mathrm{m} / \mathrm{z}:[\mathrm{M}+]$, calcd for $\mathrm{C}_{23} \mathrm{H}_{23} \mathrm{NO}_{4}, 377.1627$; found, 377.1610 .

Recovery and regeneration of $6 \boldsymbol{R}$

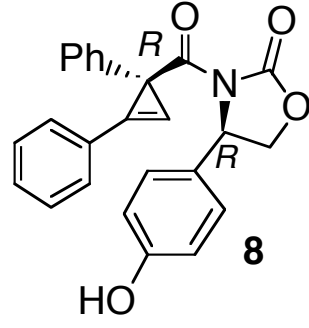

Reduction

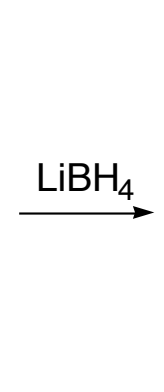

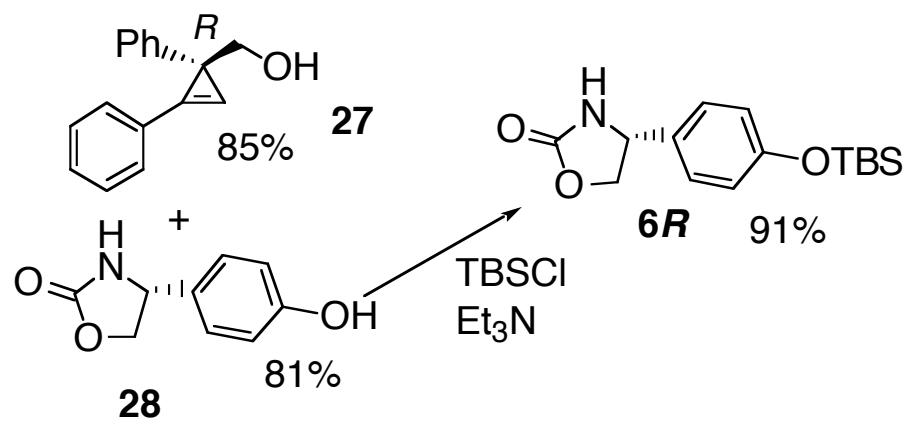
hydroxyphenyl)oxazolidinone $(\mathbf{8})$. THF $(30 \mathrm{~mL})$ was added to a dry $50 \mathrm{~mL}$ round bottomed flask that contained $\mathbf{8}(200 \mathrm{mg}, 0.50 \mathrm{mmol})$. The mixture was cooled in an ice bath, flushed with nitrogen and allowed to stir. Methanol (40 $\mu \mathrm{L}, 1.0 \mathrm{mmol})$ and $\mathrm{LiBH}_{4}$ (2.0 mmol, $2.0 \mathrm{~mL}$ of a 2.0 M solution in THF) were sequentially added, the ice bath was removed, and the mixture was allowed to stir for $4 \mathrm{~h}$ while the temperature gradually increased to rt. The reaction mixture was quenched by sat. $\mathrm{NH}_{4} \mathrm{Cl}(\mathrm{aq})$ and extracted with ether $(3 \times 30 \mathrm{~mL})$. The organics were combined, dried $\left(\mathrm{Na}_{2} \mathrm{SO}_{4}\right)$ filtered, and concentrated. The residue was chromatographed on silica gel (with a gradient of 20-70\% ethyl acetate in hexane) to first provide $96 \mathrm{mg}(0.43 \mathrm{mmol}, 86 \%$ yield $)$ of $(\boldsymbol{R})-(-)-27$, followed by to get $73 \mathrm{mg}$ ( $0.41 \mathrm{mmol}, 81 \%$ yield $)$ of $\mathbf{2 8}$

(3R)-1,3-Diphenyl-3-hydroxymethylcycloprop-1-ene $[\boldsymbol{R}-(-)-27]:[\alpha]_{\mathrm{D}}-18.7^{\circ}$ (c 0.96 , THF) $\left(\right.$ lit $\left.^{1} 18.9^{\circ}\right)$. Spectral data were identical to those already published. ${ }^{1}$

(4R)-4-(4-hydroxyphenyl)oxazolidinone (28): a white solid, $\mathrm{mp}=156-158{ }^{\circ} \mathrm{C},[\alpha]_{\mathrm{D}}$ $-34.0^{\circ}$ (c 0.98, THF). ${ }^{1} \mathrm{H}$ NMR $\left(\mathrm{CDCl}_{3}, 400 \mathrm{MHz}, \delta\right): 9.48$ (s, 1H), 8.03 (s, 1H), 7.11 (d, $\mathrm{J}=8.2 \mathrm{~Hz}, 2 \mathrm{H}), 6.74(\mathrm{~d}, \mathrm{~J}=8.2 \mathrm{~Hz}, 2 \mathrm{H}), 4.78(\mathrm{t}, \mathrm{J}=7.6 \mathrm{~Hz}, 1 \mathrm{H}), 4.56(\mathrm{t}, \mathrm{J}=8.5 \mathrm{~Hz}, 1 \mathrm{H}), 3.901$ $(\mathrm{t}, \mathrm{J}=8.6 \mathrm{~Hz}, 1 \mathrm{H}) ;{ }^{13} \mathrm{C}$ NMR (DMSO- $\left.d_{6}, 90 \mathrm{MHz}, \delta\right): 159.8(\mathrm{u}), 158.1(\mathrm{u}), 131.9(\mathrm{u})$, 128.3(dn), 116.3(dn), 72.5(u), 55.7(dn); IR(neat, $\left.\mathrm{cm}^{-1}\right): 3395,2982,1731,1601,1514$, 1239, 1029; HRMS-CI( $\left.\mathrm{NH}_{3}\right)$ m/z: [M+H] calcd for $\mathrm{C}_{9} \mathrm{H}_{10} \mathrm{O}, 180.0661$; found, 180.0659 .

Regeneration of $\mathbf{6}(\boldsymbol{R})$ : To a dry $50 \mathrm{~mL}$ round bottomed flask was added sequentially: 28 (179mg, $1.0 \mathrm{mmol})$, $t$-butyldimethylsilylchloride $(151 \mathrm{mg}, 1.0 \mathrm{mmol})$, triethylamine (202mg, 2.0mmol) and $\mathrm{CH}_{2} \mathrm{Cl}_{2}(20 \mathrm{~mL})$. The mixture was stirred at r.t. for $16 \mathrm{~h}$. Water $(20 \mathrm{~mL})$ was then added, and the organic phase was separated, dried $\left(\mathrm{Na}_{2} \mathrm{SO}_{4}\right)$, filtered, 
and concentrated. The residue was chromatographed on silica gel (using a gradient of 20$30 \%$ ethyl acetate in hexane) to provide $267 \mathrm{mg}(0.91 \mathrm{mmol}, 91 \%)$ of a white solid, $\mathrm{mp}$ $=142-143^{\circ} \mathrm{C},[\alpha]_{\mathrm{D}}-37.6^{\circ}(c 1.05, \mathrm{THF})$. Spectral characteristics were identical to those listed above.

\section{Computational details}

Quantum chemistry calculations were carried out using the Gaussian98 program system $^{2}$ utilizing gradient geometry optimization. ${ }^{3}$ All geometries were fully optimized using the B3LYP functional ${ }^{4,5}$ with the $6-31+\mathrm{G}(\mathrm{d}, \mathrm{p})$ basis set. Vibrational frequency calculations were performed to characterize the stationary points as either minima or transition structures (first-order saddle point).

\section{$\mathbf{S}_{\mathrm{cy}} \mathbf{S}_{\mathrm{ox}}$ Transition State \\ B3LYP/6-31+G(d,p) \\ $\mathrm{E}=-1598.56881$ a.u. \\ Im.Freq.=-171.8796cm-1}

$62.359239-0.181813-1.478928$

$61.5067090 .466255-2.218857$

$61.2831490 .600787-0.737371$

$61.6476911 .902563-0.050373$

62.2832611 .9341271 .204917

62.6277983 .1484321 .808782

62.3512714 .3577951 .163787

$61.7420694 .339325-0.092242$

$61.4003693 .124327-0.690094$

$63.511665-1.040822-1.318478$

$64.263931-1.431571-2.443279$

$65.372705-2.261264-2.294815$

$65.747574-2.714098-1.024187$

$65.005953-2.3328150 .09733$

$63.895886-1.50027-0.047104$

$3-0.1975611 .7096862 .264336$

$6-2.7687272 .247953-1.864442$

$6-2.2298860 .811016-1.610434$

$6-3.336656-0.227002-1.467463$

$6-3.41091-1.307819-2.353207$

$7-1.4332191 .000631-0.39427$

$6-1.7279552 .1751640 .151352$

$8-1.3324122 .6542951 .23773$

$8-2.6045152 .924572-0.604911$

$60.14141-0.260051-0.175964$

$8-0.211641-1.304337-0.666268$ 


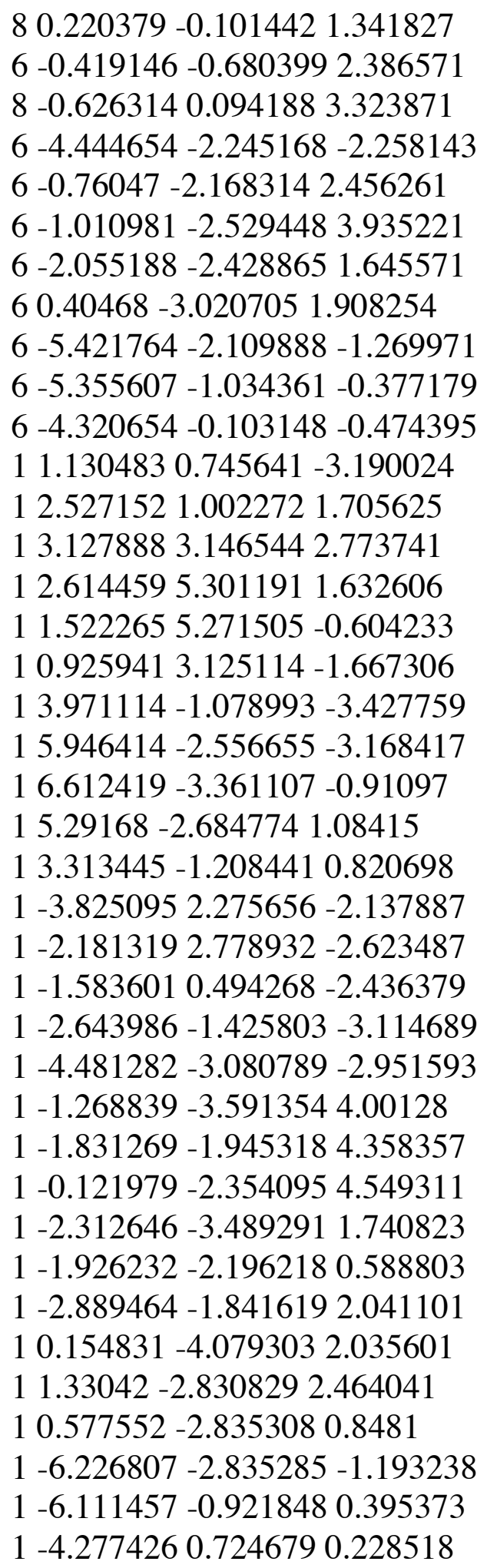

Experimental Section Page-15 


$$
\begin{aligned}
& \mathbf{R}_{\text {cy }} \mathbf{S}_{\text {ox }} \text { Transition State } \\
& \mathrm{E}=-1598.56712 \text { a.u. } \\
& \text { Im.Freq. }=-172.4619 \mathrm{~cm}-1 \\
& 6-2.093902-0.48148-1.122149 \\
& 6-1.356705-0.192325-2.156623 \\
& 6-1.2202510 .739435-0.982869 \\
& 6-1.8343742 .131405-1.062155 \\
& 6-1.075963 .253586-1.434205 \\
& 6-1.6743594 .50951-1.557692 \\
& 6-3.0405284 .671099-1.3108 \\
& 6-3.8050863 .562034-0.942946 \\
& 6-3.2054552 .30568-0.821626 \\
& 6-3.042566-1.358967-0.462676 \\
& 6-3.542972-2.489247-1.139115 \\
& 6-4.445295-3.344275-0.512193 \\
& 6-4.861264-3.0929070 .801341 \\
& 6-4.36949-1.9768751 .483294 \\
& 6-3.466968-1.1123460 .857541 \\
& 3-0.671638-2.1187961 .192847 \\
& 62.158324-1.846089-2.755283 \\
& 62.140646-0.593302-1.826253 \\
& 63.494234-0.312605-1.185 \\
& 64.2588430 .78303-1.602899 \\
& 71.090732-0.935337-0.867538 \\
& 60.775707-2.217738-0.994437 \\
& 8-0.017528-2.892212-0.295592 \\
& 81.434434-2.852695-2.023803 \\
& 60.0453040 .70742-0.103029 \\
& 80.8813141 .565258-0.031787 \\
& 8-0.403276-0.0453071 .181729 \\
& 60.2009-0.2291762 .379048 \\
& 80.082818-1.3720132 .831883 \\
& 65.5263261 .022602-1.06305 \\
& 60.876940 .8946643 .164639 \\
& 66.0468530 .162429-0.093749 \\
& 65.289505-0.9336290 .333229 \\
& 64.023213-1.166585-0.206272 \\
& 60.8468280 .5106554 .659935 \\
& 62.3536391 .000172 .702371 \\
& 60.1430722 .2372242 .965567 \\
& 1-1.02149-0.374243-3.165019 \\
& 1-0.0130563 .144531-1.6158
\end{aligned}
$$


$1-1.0691525 .36365-1.848821$

$1-3.5032395 .649238-1.40589$

$1-4.8684293 .671038-0.74836$

$1-3.8157361 .451514-0.544922$

$1-3.206781-2.693696-2.150656$

$1-4.820415-4.213716-1.043774$

$1-5.56261-3.763861 .288227$

$1-4.69077-1.7741572 .500778$

$1-3.09215-0.2386511 .381039$

$13.163267-2.221143-2.958348$

$11.638438-1.665418-3.703752$

$11.8536790 .297468-2.398781$

$13.8544211 .462882-2.349388$

$16.1014421 .882357-1.395611$

17.0307120 .3453260 .328862

$15.685437-1.6063991 .089162$

$13.437661-2.013290 .140815$

11.3452511 .2959355 .237331

$11.359613-0.4359374 .842937$

$1-0.1794490 .4153915 .029566$

12.8443241 .7830263 .290811

12.4309261 .2579281 .646135

12.8850350 .0598062 .878151

10.613552 .9923543 .603974

$1-0.9096642 .1630883 .260492$

10.195932 .5841081 .933943 


\section{Archive File for the RcySox Transition State:}

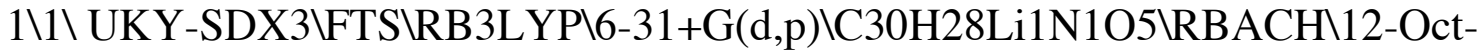
$2003 \backslash 0$

॥ FREQ=NORAMAN SCF(MAXCYC=750)

OPT $=($ TS,NOEIGEN,READFC,GDIIS) $\operatorname{IOP}(1 / 8$ =2) GEOM=CHECK GUESS=READ B3LYP/6-

$31+\mathrm{G}(\mathrm{D}, \mathrm{P}) \backslash \mathrm{Ph} \_\mathrm{RS} \_$down $\backslash \backslash 0,1 \backslash \mathrm{C},-2.0940$

758011,-0.4794561397,-1.1226919119\C,-1.3563881254,-0.1908051074,-2.15 $69571407 \backslash \mathrm{C},-1.2193709356,0.7406619378,-0.9830369186 \backslash \mathrm{C},-1.8322388052,2$. $13318853,-1.0622587059 \backslash \mathrm{C},-1.0727432527,3.2547491931,-1.4339747375 \backslash \mathrm{C},-1$ $.6699980879,4.5112231076,-1.5574134666 \backslash \mathrm{C},-3.0360788763,4.6739896933,-1$ $.3108065917 \backslash \mathrm{C},-3.8017057611,3.5655510857,-0.9432868723 \backslash \mathrm{C},-3.2032188491$ ,2.3086473919,-0.8220142133\C,-3.0436680115,-1.3561946146,-0.463561379 $1 \backslash \mathrm{C},-3.5449250504,-2.4859315808,-1.14027819 \backslash \mathrm{C},-4.4481497165,-3.3402487$ $032,-0.5136843862 \backslash C,-4.8641922061,-3.0887016497,0.7997925104 \backslash C,-4.3715$ $806993,-1.9732061687,1.4820188244 \backslash \mathrm{C},-3.4681493262,-1.1093892534,0.8565$ 960108\Li,-0.6737909383,-2.118371438,1.192386863\C,2.1573054007,-1.847 $6058664,-2.7550638191 \backslash \mathrm{C}, 2.1405307721,-0.5949387467,-1.8258552007 \backslash \mathrm{C}, 3.4$ $942224349,-0.3155380968,-1.184255773 \backslash \mathrm{C}, 4.2598992445,0.7794780275,-1.60$ $18224562 \backslash N, 1.0900962259,-0.9361800695,-0.8674279284 \backslash C, 0.7739604963,-2$. $2182814933,-0.9945843539 \backslash \mathrm{O},-0.0200318372,-2.892152355,-0.2960173739 \backslash \mathrm{O}$, $1.4323561051,-2.8536747966,-2.0238941941 \backslash \mathrm{C}, 0.0459557002,0.7073943212,-$ $0.1029160998 \backslash \mathrm{O}, 0.8827123094,1.5644783901,-0.0313595703 \backslash \mathrm{O},-0.403583759$, $-0.0451207241,1.1816310281 \backslash \mathrm{C}, 0.2001578931,-0.2297003336,2.3790597309 \backslash \mathrm{O}$ $, 0.0809587008,-1.3724975594,2.83170186 \backslash C, 5.527472912,1.0178440273,-1.0$ 616527846\C,0.8770193429,0.8934237601,3.1649675851\C,6.0470161628,0.15 70686968,-0.0923585079\C,5.2885978043,-0.9383787036,0.3342884479\C,4.0 $222213382,-1.1701304087,-0.2055328719 \backslash \mathrm{C}, 0.8462283844,0.509224877,4.660$ $2005509 \backslash \mathrm{C}, 2.3539162781,0.9976849305,2.7030488472 \backslash \mathrm{C}, 0.1443897274,2.2366$ $649849,2.965924978 \backslash \mathrm{H},-1.0211062388,-0.3728748415,-3.165304574 \backslash \mathrm{H},-0.009$ $8962168,3.1447760363,-1.61534522 \backslash \mathrm{H},-1.0639663055,5.3648672414,-1.84828$ $19599 \backslash \mathrm{H},-3.4978990531,5.6525538148,-1.4058592056 \backslash \mathrm{H},-4.864995238,3.6754$ $725956,-0.7489258412 \backslash \mathrm{H},-3.8143213203,1.4549839964,-0.5455725824 \backslash \mathrm{H},-3.2$ $086872455,-2.6905325999,-2.1517723382 \backslash H,-4.823921828,-4.2092782492,-1$. $0454764457 \mathrm{H},-5.5662444054,-3.7591020694,1.2864224192 \backslash \mathrm{H},-4.6929103985$, $-1.7703508001,2.4994592619 \backslash \mathrm{H},-3.092673381,-0.2361030296,1.3803054053 \backslash \mathrm{H}$ ,3.1619610037,-2.2235233984,-2.9579561166\H,1.6377945934,-1.6663350264 ,-3.7036242679\Н,1.8544851697,0.2961689004,-2.3983189624\Н,3.856250314 $3,1.4597968114,-2.3483037929 \backslash \mathrm{H}, 6.1034283955,1.877136679,-1.3939577862 \backslash$ H,7.0309420832,0.3390297785,0.3305008685\Н,5.6837603074,-1.611609992,1 $.0902125572 \backslash \mathrm{H}, 3.4358386038,-2.0163651442,0.1412982699 \backslash \mathrm{H}, 1.3452176301,1$ $.2939770635,5.2378235867 \backslash \mathrm{H}, 1.3581300452,-0.4378488794,4.8431810334 \backslash \mathrm{H},-$ 
$0.1802167738,0.4148192353,5.0295856237 \mathrm{H}, 2.8451630807,1.7800184967,3.2$ $917128566 \backslash \mathrm{H}, 2.431670461,1.2555280957,1.6468671778 \backslash \mathrm{H}, 2.8844357283,0.056$ $8233289,2.8788112607 \backslash \mathrm{H}, 0.6153947479,2.9912831504,3.6045484287 \backslash \mathrm{H},-0.908$ $4784762,2.1634219901,3.2606012563 \backslash \mathrm{H}, 0.1977894731,2.5836517885,1.934363$ 3697\IVersion=HP-PARisc-HPUX-G98RevA.11 $\mathrm{HF}=-$

1598.5671207\RMSD=5.200e-0

9\RMSF=2.117e-06\ipole $=-0.9649648,0.4492814,0.2187534 \backslash \mathrm{PG}=\mathrm{C} 01$

$[\mathrm{X}(\mathrm{C} 30 \mathrm{H} 2$

8Li1N1O5)]川@ 


\section{Archive File for the ScySox Transition State:}

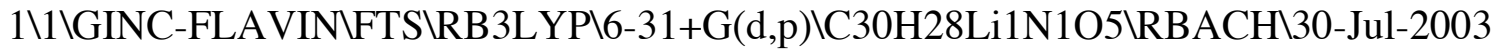
$\backslash 0 \backslash$ \# OPT=(TS,NOEIGEN,READFC,GDIIS,MODREDUN) IOP $(1 / 8=1)$

GEOM=CHECK RB3

LYP/6-31+G(D,P) SCF(MAXCYC=250)\Ph_SS_up \0,1\C,2.3917696913,-0.06490 $9502,-1.4357993699 \backslash \mathrm{C}, 1.537437421,0.5897534542,-2.1678022813 \backslash \mathrm{C}, 1.281392$ $278,0.6610203327,-0.6871807791 \backslash \mathrm{C}, 1.5985023786,1.9439033344,0.056546369$ $\backslash \mathrm{C}, 2.2082468208,1.9426891508,1.3249738062 \backslash \mathrm{C}, 2.5092641836,3.1409184022$, 1.9818833266\C,2.2142179714,4.3670709777,1.3781086246\C,1.630503406,4. $3820559336,0.1099884348 \backslash \mathrm{C}, 1.3322219999,3.1829729244,-0.5408820932 \backslash \mathrm{C}, 3$. $5626939261,-0.9002560793,-1.2846150522 \backslash C, 4.3467948375,-1.2280196104,-2$ $.4077897896 \backslash \mathrm{C}, 5.4735669304,-2.0347138723,-2.2683591818 \backslash \mathrm{C}, 5.8350006345$, $-2.5269568971,-1.0085395366 \backslash C, 5.0618486334,-2.2082541535,0.1112120832 \backslash$ C,3.9336313849,-1.3989001366,-0.0241110248\Li,-0.286267428,1.615005375 7,2.3235931924\C,-2.7888758397,2.2483106643,-1.8337273579\C,-2.2180733 $277,0.8165979951,-1.6238712018 \backslash \mathrm{C},-3.3001665994,-0.2536814469,-1.543632$ $0105 \backslash \mathrm{C},-3.3289621634,-1.3008309146,-2.4714627528 \backslash \mathrm{N},-1.4506442899,0.978$ $8338426,-0.3851536292 \backslash \mathrm{C},-1.7863867376,2.123524324,0.1988514147 \backslash \mathrm{O},-1.42$ $48212167,2.5699203043,1.310713707 \backslash \mathrm{O},-2.6670406636,2.8794484006,-0.5460$ $855397 \backslash \mathrm{C}, 0.1515328032,-0.2493882311,-0.1828380437 \backslash \mathrm{O},-0.1646310213,-1.2$ $824021526,-0.7199587482 \backslash \mathrm{O}, 0.1965187651,-0.147873251,1.3412200981 \backslash \mathrm{C},-0$. $4481966141,-0.7828375647,2.3496023861 \backslash \mathrm{O},-0.6937505977,-0.0506666257,3$. $3114049798 \backslash \mathrm{C},-4.3397351308,-2.2668535069,-2.4337229903 \backslash \mathrm{C},-0.7521495453$ $,-2.2804458527,2.3551974693 \backslash \mathrm{C},-1.022235594,-2.7048946988,3.8137557709 \backslash$ C,-2.0234964384,-2.5418005185,1.5085753985\C,0.445245767,-3.0813651397 $, 1.7991111844 \backslash \mathrm{C},-5.3392558278,-2.1946346805,-1.4614719619 \backslash \mathrm{C},-5.3185541$ 792,-1.1532471298,-0.5269695874\C,-4.3063706163,-0.1932400542,-0.56706 94026\H,1.1732574203,0.8970762957,-3.1351155504\Н,2.4663177575,0.99852 $88756,1.7945527692 \backslash \mathrm{H}, 2.9901726309,3.1141513294,2.9561774844 \backslash \mathrm{H}, 2.443603$ $7301,5.297877595,1.8880460623 \backslash \mathrm{H}, 1.3967192485,5.3275851795,-0.370353565$ $6 \backslash \mathrm{H}, 0.877230432,3.2097610073,-1.5269300313 \backslash \mathrm{H}, 4.0643444556,-0.844969370$ 2,-3.3838779419\Н,6.0717974996,-2.2814600656,-3.1406063043\Н,6.7139307 $777,-3.1559267004,-0.9023605421 \backslash \mathrm{H}, 5.3371503127,-2.5909559945,1.0895152$ $028 \backslash \mathrm{H}, 3.3268796861,-1.1557070781,0.8420246314 \backslash \mathrm{H},-3.8400293272,2.260042$ $056,-2.1276541736 \backslash \mathrm{H},-2.2006210779,2.8229494375,-2.5595940653 \backslash \mathrm{H},-1.5476$ $882577,0.5484932042,-2.4478257694 \backslash \mathrm{H},-2.5444201526,-1.3698534076,-3.220$ $9147334 \backslash \mathrm{H},-4.3410595714,-3.0755860417,-3.1592755041 \backslash \mathrm{H},-1.2537360029,-3$ $.7747107433,3.8337615265 \backslash \mathrm{H},-1.8655442297,-2.1584329813,4.2419333956 \backslash \mathrm{H}$, $-0.1503175251,-2.5312147944,4.4523279802 \backslash \mathrm{H},-2.2552085702,-3.6112614644$ , 1.5578104846\H,-1.8798686063,-2.2651443424,0.4643794115\H,-2.88032376 $77,-1.9915070503,1.9090026298 \backslash \mathrm{H}, 0.2204599918,-4.1500540569,1.880638994$ $2 \backslash \mathrm{H}, 1.3546513852,-2.8899925639,2.3807499523 \backslash \mathrm{H}, 0.6340569716,-2.85067351$ $22,0.7506297313 \backslash \mathrm{H},-6.1265815678,-2.9424715214,-1.4292086251 \mathrm{H},-6.09210$ $35515,-1.0898408634,0.2335606384 \backslash \mathrm{H},-4.2984346259,0.6075061181,0.167774$ 6595\Version=SGI64-G98RevA.11\HF=-1598.5688101\RMSD=3.147e-09\RMSF=4. 
567e-05\Dipole $=1.532564,-0.5447861,-0.3270389 \backslash \mathrm{PG}=\mathrm{C} 01[\mathrm{X}(\mathrm{C} 30 \mathrm{H} 28 \mathrm{Li1N} 1 \mathrm{O})$

]1@

\section{References:}

(1) Liao, L.-a.; Zhang, F.; Yan, N.; Golen, J. a.; Fox, J. M. Tetrahedron in press.

(2) Frisch, M. J.; Trucks, G. W.; Schlegel, H. B.; Scuseria, G. E.; Robb, M. A.; Cheeseman, J. R.; Zakrzewski, V. G.; Montgomery, J. A.; Stratmann, R. E.; Burant, J. C.; Dapprich, S.; Millam, J. M.; Daniels, A. D.; Kudin, K. N.; Strain, M. C.; Farkas, O.; Tomasi, J.; Barone,V.; Cossi, M.; Cammi,R.; Mennucci, B.; Pomelli, C.; Adamo, C.; Clifford, S.; Ochterski, J.; Petersson, G. A.; Ayala, P. Y.; Cui, Q.; Morokuma, K.; Malick, D. K.; Rabuck, A. D.; Raghavachari, K.; Foresman, J. B.; Cioslowski, J.; Ortiz, J. V.; Baboul, A. G.; Stefanov, B. B.; Liu, G.; Liashenko, A.; Piskorz, P.; Komaromi, I.; Gomperts, R.; Martin, R. L.; Fox, D. J.; Keith, T.; Al-Laham, M. A.; Peng, C. Y.; Nanayakkara, A.; Gonzalez, C.; Challacombe, M.; Gill, P. M. W; Johnson, B.; Chen, W.; Wong, M. W.; Andres, J. L.; Gonzalez, C.; Head-Gordon, M.; Replogle, E. S.; Pople, J. A. Gaussian 98, Revision A.7, Gaussian, Inc., Pittsburgh PA, 1998.

(3) (a) Schlegel, H. B. J. Comput. Chem. 1982, 3, 214. (b) Schlegel, H. B. Adv. Chem. Phys. 1987, 67(Pt. 1), 249. (c) Schlegel, H. B. In Modern Electronic Structure Theory, Yarkony, D. R., Ed.; World Scientific: Singapore, 1995; p 459.

(4) (a) Becke, A. D. Phys. Rev. A 1988, 38, 3098. (b) Lee, C.; Yang, W. and Parr, R. G. Phys. Rev. B 1988, 37, 785.

(5) (a) Becke, A. D. J. Chem. Phys. 1993, 98, 5648. (b) Stevens, P. J.; Devlin, F. J.; Chabalowski, C. F. and Frisch, M. J. J. Phys. Chem. 1994, 98, 11623. 